\title{
Global regularity for the minimal surface equation in Minkowskian geometry
}

\author{
Atanas Stefanov \\ Communicated by Christopher D. Sogge
}

\begin{abstract}
We study the minimal surface equation in Minkowskian geometry in $\mathbb{R}^{n} \times \mathbb{R}_{+}^{1}$, which is a well-known quasilinear wave equation. The classical result of Lindblad, [10], establishes global existence of small and smooth solutions (i.e. global regularity), provided the initial data is small, compactly supported and very smooth. In the present paper, we achieve more precise results. We show that, at least when $n \geq 4$ (or $n=3$, but with radial data), it is enough to assume the smallness of some scale invariant quantities, involving (unweighted) Sobolev norms only.

In the $3 \mathrm{D}$ case, such a proof fails as a consequence of the well-known Strichartz inequality "missing endpoint" and one has instead slightly weaker results, which requires smallness of the data in certain $W^{s, p}, p<2$, spaces.

In the $2 \mathrm{D}$ case, this fails as well, since the free solution of the $2 \mathrm{D}$ wave equation fails to be square integrable, but only belongs to $L^{2, \infty}$, another failure by an endpoint. Thus, an important question is left open: Can one prove global regularity for the $2 \mathrm{D}$ minimal surface equation, assuming smallness in unweighted Sobolev spaces only?
\end{abstract}

Keywords. Minimal surface equation, small solutions, global regularity, quasilinear wave equations.

2000 Mathematics Subject Classification. Primary 35L70; secondary 58E12, 58J45, 49Q15.

\section{Introduction}

In this paper we shall be concerned with the minimal surface equation, where the background metric is the Lorentzian metric. This is a well-known model, whose derivation we discuss briefly.

Consider the usual Euclidean space $\mathbb{R}^{n+2}$ endowed with a Riemannian metric given by the symmetric metric tensor

$$
R=g^{i j}(x) d x_{i} d x_{j}
$$

Supported in part by NSF-DMS 0701802 and 0908802. 
To simplify our considerations, take a surface in the form $x_{n+1}=\phi\left(x_{0}, \ldots, x_{n}\right)$ and $g^{(n+1) i}=\delta_{(n+1) i}$. Compute the surface area by

$$
S(\phi)=\int_{\mathbb{R}^{n+1}} \sqrt{1+\sum_{i, j=0}^{n} g^{i j}(x) \partial_{i} \phi \partial_{j} \phi} d x=\int_{\mathbb{R}^{n+1}} L(\dot{\phi}) d x .
$$

The problem of finding the minimal surfaces embedded in $\mathbb{R}^{n+2}$ with a metric tensor $R$ reduces to minimizing the functional $S(\phi)$, when restricted to some class of admissible functions $\phi$. This is, of course, the standard setup of calculus of variations, and if one makes the usual assumptions on smoothness (as we shall do later on) the minimizers must satisfy the Euler-Lagrange equation

$$
\partial^{i}\left(\frac{\partial L}{\partial \dot{\phi}_{i}}\right)-\frac{\partial L}{\partial \phi}=0
$$

Since $\frac{\partial L}{\partial \phi}=0$ and

$$
\frac{\partial L}{\partial \dot{\phi}_{i}}=\frac{g_{i}^{j} \partial_{j} \phi}{2 \sqrt{1+g^{k m}(x) \partial_{k} \phi \partial_{m} \phi}},
$$

we get the minimal surface equation

$$
\partial^{i} \frac{g_{i}^{j} \partial_{j} \phi}{\sqrt{1+g^{k m}(x) \partial_{k} \phi \partial_{m} \phi}}=0 .
$$

In the Euclidean case, one has $g^{i j}=\delta_{i j}$ and (1.1) takes the form

$$
\partial^{j} \frac{\partial_{j} \phi}{\sqrt{1+|\nabla \phi|^{2}}}=0
$$

or

$$
\operatorname{div}\left(\frac{\nabla \phi}{\sqrt{1+|\nabla \phi|^{2}}}\right)=0,
$$

which has been extensively studied by many authors. We will not even attempt to give an account of these studies, since this is outside the scope of this paper.

In the Minkowski metric $R=\operatorname{diag}(-1,1, \ldots, 1)$ setting, with the usual assignment of $x_{0}$ as the "time" variable and $\left(x_{1}, \ldots, x_{n}\right)$ as the "space" vector, equation (1.1) becomes

$$
\partial_{t} \frac{\partial_{t} \phi}{\sqrt{1+|\nabla \phi|^{2}-\left(\partial_{t} \phi\right)^{2}}}-\partial^{j} \frac{\partial_{j} \phi}{\sqrt{1+|\nabla \phi|^{2}-\left(\partial_{t} \phi\right)^{2}}}=0 .
$$


Note that the Langrangean density $L$ is not well defined, unless the function $\phi$ has the property $\left(\partial_{t} \phi\right)^{2}-|\nabla \phi|^{2}<1$.

We now describe the current and latest results in the study of existence and uniqueness for solutions of the minimal surface equation (1.2). In order to write the equation in a more tractable form, introduce the bilinear operator $Q(u, v)$ defined by

$$
Q(u, v)=\partial_{t} u \partial_{t} v-\partial^{i} u \partial_{i} v .
$$

We can now rewrite equation (1.2) (assuming enough smoothness for the unknown function $\phi$ ) as

$$
\frac{\partial_{t}^{2} \phi-\Delta \phi}{\sqrt{1-Q(\phi, \phi)}}+\frac{\phi_{t} \partial_{t} Q(\phi, \phi)-\partial^{j} \phi \partial_{j} Q(\phi, \phi)}{(1-Q(\phi, \phi))^{3 / 2}}=0 .
$$

Taking common denominators and taking into account

$$
\phi_{t} \partial_{t} Q(\phi, \phi)-\partial^{j} \phi \partial_{j} Q(\phi, \phi)=Q(\phi, Q(\phi, \phi))
$$

leads us to consider the following Cauchy problem:

$$
\left\{\begin{array}{l}
\square \phi-Q(\phi, \phi) \square \phi=-Q(\phi, Q(\phi, \phi)) / 2, \\
\phi(x, 0)=f(x), \\
\phi_{t}(x, 0)=g(x) .
\end{array}\right.
$$

One should note that (1.3) is not guaranteed to be a hyperbolic equation, if $\|\nabla \phi\|_{L^{\infty}}$ is not small.

A local well-posedness theory for (1.3) (without smallness assumptions) can be built from the methods developed in [5], see also [13], [2]. Basically, making use of some (mild) symmetries of the system, one shows that energy estimates close, at least for short time, when $(f, g) \in H^{n / 2+2} \times H^{n / 2+1}$. In order to set a clear reference on how good such a result is, we discuss the scaling of (1.3). More specifically, if $\phi$ is a solution of (1.3), then $\phi_{\lambda}(x, t)=\lambda^{-1} \phi(\lambda x, \lambda t)$ is a solution of (1.3) as well with initial data $\left(\lambda^{-1} f(\lambda \cdot), g(\lambda \cdot)\right)$. This dictates that the critical Sobolev index for the equation (1.3) is $s_{c}=n / 2+1$.

That is, we expect to have ill-posedness for (1.3), whenever $(f, g) \in\left(H^{s} \times\right.$ $H^{s-1}$ ) and $s<n / 2+1$, where $H^{s}$ are the standard Sobolev spaces. On the other hand, this is just a lower bound and the well-posedness theory for the Cauchy problem for this quasilinear wave equation may well get into trouble for values of $s$ nontrivially bigger than the scaling index.

For global existence results, Lindblad [10] has shown that the problem (1.3) with initial data $(\varepsilon f, \varepsilon g)$ has global solutions, provided $0<\varepsilon \ll 1$ and $f, g \in$ 
$C^{\infty}\left(\mathbb{R}^{n}\right), n \geq 2$. Lindblad actually provides two proofs. In each of the proofs, he heavily uses the structure of the bilinear operator $Q$ as well as the conformal properties of (1.3). In fact, his first proof contains more information than stated above. Namely, his method provides global solutions for data $(\varepsilon f, \varepsilon g)$, which is in the class $H^{2 n+2,2 n+2}\left(\mathbb{R}^{n}\right) \times H^{2 n+1,2 n+1}$. Here $H^{m, l}$ is the weighted Sobolev space $\left\{f: \int\left(1+|x|^{l}\right)^{2}\left[|f(x)|^{2}+\left|\partial^{m} f(x)\right|^{2}\right] d x<\infty\right\}$. The proof essentially relies on the $H^{1}$ energy and decay estimates (similar to those in [5]), applied to the wave equations satisfied by $\Gamma^{I} \phi$, where $\Gamma^{I}$ are products of $|I|$ copies of the Lorentzian group generators $\Gamma_{j k}=x_{j} \partial_{k}-x_{k} \partial_{j},(j, k) \neq(0,0)$, $\Gamma_{00}=\sum_{j=0}^{n} x_{j} \partial_{j}$.

Subsequent developments to this approach led to the publications [3] - for the related Born-Infeld model - and [2] - for minimal surfaces of general codimension. These are all "small data" results, similar in spirit to the work of Lindblad, [10]. We would like to specifically mention another line of investigation, in which the smallness assumption was removed. In [9], the authors build a smooth classical solution, under a condition on the initial data, which they show to be necessary for the global existence. That is, this condition guarantees the validity of $1-Q(\phi, \phi)>0$ (which appears under square root in the equation) and global existence and conversely, if this condition is violated by the initial data, singularities will emerge in finite time. In follow up papers, [11] and [12], the authors consider related problems (initial-boundary value problems etc.) where similar necessary and sufficient conditions for global existence were found.

We would like to point out that in these geometric works, [9], [11] and [12], the emphasis is on global in time, classical solution, without particular regard for decay at spatial infinity. On the other hand, the approach in [10] and subsequently [3], [2], the authors employ Klainerman's vector field approach and as a consequence they obtain energy estimates as well as time-decay estimates for various $L_{x}^{\infty}$ norms of (angular) derivatives of $\phi$.

In this paper, we study (1.3) with small data in (unweighted) Sobolev spaces. The goal is to produce global solutions with minimal regularity assumptions on the small data, which can be readily compared to the critical index. Moreover, at least in the high-dimensional case $n \geq 4$, we will be able to present the smallness condition in a scale invariant form, which is a natural setup for this problem.

Question. Under what (minimal) Sobolev regularity conditions on the (small) data $(f, g)$, one has global existence and persistence of the solutions to (1.3)?

Since our interest is in global solutions, and by the results in [9], we will need to ensure the positivity of $1+|\nabla \phi|^{2}-\left(\partial_{t} \phi\right)^{2}$. In particular, this will follow, if we require that $\|\nabla \phi\|_{L^{\infty}} \ll 1$, which we will do henceforth. 
Let us justify this by the following argument. According to the postulates of special relativity (which is modeled in our case by $\mathbb{R}^{n+1}$ endowed with the Minkowski metric), every object has a velocity less than the speed of light, in our units every velocity is less than one. This implies the causality principle, which informally states that every trajectory through a fixed point $P$ must lie in a cone of aperture one centered at $P$. Take such a trajectory on our surface and suppose $\left|\nabla \phi\left(x^{0}\right)\right|>1$. Then, there exists a direction $\xi$, so that for sufficiently small $t$, one has $\left|\phi\left(x^{0}+y \xi\right)-\phi\left(x^{0}\right)\right|>|y|$. That implies that such a trajectory leaves the light cone, in direct violation of the causality principle. Equivalently, the speed of the point $P$, in the direction of $\xi$, will be larger than the speed of light. Thus, it makes sense to require that $\phi:\left|\nabla \phi\left(x^{0}\right)\right| \leq 1$ and in fact is physically relevant to consider only $\left|\nabla \phi\left(x^{0}\right)\right| \ll 1$, since most velocities ought to be negligible with respect to the speed of light.

Our findings are summarized in the following theorems.

Theorem 1.1. Let $n \geq 4$ or $n=3$ and the data $(f, g)$ is radial. Let also $0<\mu<$ $(n-1) / 2$. Then, there exists $\kappa=\kappa(n, \mu)>0$, so that the Cauchy problem (1.3) has a global solution, whenever

$$
f \in \dot{H}^{n / 2+5 / 2+\mu} \cap \dot{H}^{n / 2-1 / 2-\mu}, g \in \dot{H}^{n / 2+3 / 2+\mu} \cap \dot{H}^{n / 2-3 / 2-\mu}
$$

and

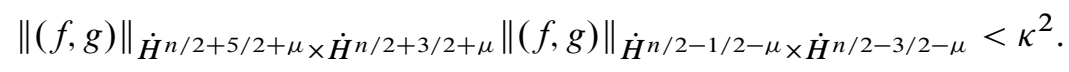

Moreover, there are the persistence estimates, namely for all $s>1$,

$$
\|\phi\|_{L^{\infty}(0, \infty) \dot{H}^{s}} \leq C\|(f, g)\|_{\dot{H}^{s} \times \dot{H}^{s-1}} .
$$

Remarks. - Note that the quantity (1.4) is scale invariant.

- The energy estimates in (1.5) can be extended to $s=1$ by applying the standard Hörmander energy estimates, see Remark 3.2 (2), after Proposition 3.1 below.

We formulate the following corollary of the main result, which takes away most of the technical assumptions, while preserving the gist of the statement.

Corollary 1.2. Let $n \geq 4$ or $n=3$ and the data $(f, g)$ be radial, $\mu>0$. Then, there exists $\kappa>0$, so that the Cauchy problem (1.3) has a global solution, whenever $f \in H^{n / 2+5 / 2+\mu}, g \in H^{n / 2+3 / 2+\mu}$ and

$$
\|(f, g)\|_{H^{n / 2+5 / 2+\mu} \times H^{n / 2+3 / 2+\mu}}<\kappa .
$$


In other words, if the initial data is small in the space $H^{n / 2+5 / 2+} \times H^{n / 2+3 / 2+}$, then the solution is global and stays small. Note that this space requires the initial data to be $3 / 2+$ derivatives smoother than the lower bound provided by the scaling analysis. Then again, even the best local well-posedness result (which we have alluded to above) requires smoothness of at least $H^{n / 2+2} \times H^{n / 2+1}$. That is, our global regularity result requires $1 / 2+$ derivative more than the best available 1.w.p. result.

For the 3D case, the proof of Theorem 1.1 fails as a consequence of the wellknown failure of the Strichartz estimate at the endpoint. We do not know, whether this is due to our method of proof or it is intrinsic. We have instead the following slightly weaker result

Theorem 1.3 (3D case). Fix $4<p<\infty$. Then, there exists $\varepsilon=\varepsilon_{p}>0$, so that the Cauchy problem (1.3) has a global solution, whenever

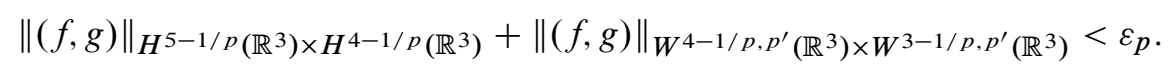

Moreover, the solution satisfies for all $0 \leq s \leq 5-1 / p, 0 \leq \alpha \leq 2+3 / p$,

$$
\begin{aligned}
& \|\phi\|_{L^{\infty}(0, \infty) H^{s}} \leq C\|(f, g)\|_{H^{s} \times H^{s-1}}, \\
& \left\||\nabla|{ }^{\alpha} \phi\right\|_{L^{2}(0, \infty) L^{p}} \leq C\left(\|(f, g)\|_{H^{5-1 / p} \times H^{4-1 / p}}\right. \\
& \left.+\|(f, g)\|_{W^{4-1 / p, p^{\prime}} \times W^{3-1 / p, p^{\prime}}}\right) .
\end{aligned}
$$

The strategy of the proof is as follows. First, we establish energy estimates for $\dot{H}^{s}, s>1$, norms - this is done in great detail in Proposition 3.1 in Section 3 below. These estimates remain valid as long as (3.1) below holds. Then, we need an additional step, which closes the argument, namely the quantities involved in (3.1) stay bounded in terms of only the relevant $\dot{H}^{s}$ norms, which we know $a$ posteriori from the fact that the energy estimates are under control.

\section{Preliminaries}

Define the Fourier transform $\mathscr{F}$ acting on a function $f \in S(S$ is the Schwartz class of test functions)

$$
\hat{f}(\xi)=\int_{\mathbb{R}^{n}} f(x) e^{-i x \xi} d x .
$$

The (homogeneous) Sobolev space $\dot{H}^{s}$ is defined as the completion of all Schwartz functions in the norm

$$
\|f\|_{\dot{H}^{s}}=\left(\int_{\mathbb{R}^{n}}|\hat{f}(\xi)|^{2}|\xi|^{2 s} d \xi\right)^{1 / 2} .
$$


We will also use the inhomogeneous version $H^{s}$, defined by the norm

$$
\|f\|_{H^{s}}=\left(\int_{\mathbb{R}^{n}}|\hat{f}(\xi)|^{2}\left(1+|\xi|^{2}\right)^{s} d \xi\right)^{1 / 2} .
$$

We analyze (1.3) via Littlewood-Paley decompositions of the solution $\phi$. Let $\psi \in C_{0}^{\infty}\left(\mathbb{R}^{n}\right)$ be such that supp $\psi \subset(0,2)$ and $\psi(\xi)=1$ for all $|\xi| \leq 1$.

Let $\varphi(\xi)=\psi(\xi)-\psi(2 \xi)$. Clearly

$$
\sum_{i \in Z} \varphi\left(2^{-i} \xi\right) \equiv 1 \quad \text { for all } \xi \neq 0
$$

which gives rise to the Littlewood-Paley operators, defined by the multipliers $\varphi\left(2^{-k} \cdot\right)$, namely

$$
\widehat{P_{k} f}(\xi):=\varphi\left(2^{-k} \xi\right) \hat{f}(\xi) .
$$

Roughly speaking, the Littlewood-Paley operators are projections with range all functions having Fourier support in the annulus $\left\{\xi: 2^{k-1} \leq|\xi| \leq 2^{k+1}\right\}$. We will very often write $f_{k}$ instead of $P_{k} f$ and $P_{<k}:=\sum_{l<k} P_{l}$. Note that

$$
f=\sum_{k \in Z} f_{k}=\lim _{k \rightarrow \infty} P_{<k} f .
$$

That is, the function $f$ is represented as a sum of (smooth) functions $f_{k}$, with mutually disjoint Fourier support. We now recall the Littlewood-Paley square function characterization of $L^{p}$ (or more generally $W^{s, p}$ spaces), namely for all $1<p<\infty$,

$$
\|f\|_{W^{s, p}} \sim\left\|\left(\sum_{k} 2^{2 k s}\left|f_{k}\right|^{2}\right)^{1 / 2}\right\|_{L^{p}} .
$$

Due to (2.1) and the inclusion $l^{p} \subset l^{q}$ for all $1 \leq p<q \leq \infty$, we obtain the useful formulae

$$
\begin{aligned}
\|f\|_{W^{s, p}} & \leq C\left(\sum_{k} 2^{2 k s}\left\|f_{k}\right\|_{L^{p}}^{2}\right)^{1 / 2}, & & 2 \leq p \leq \infty, \\
\left(\sum_{k} 2^{2 k s}\left\|f_{k}\right\|_{L^{p}}^{2}\right)^{1 / 2} & \leq C\|f\|_{W^{s, p},}, & & 1 \leq p \leq 2 .
\end{aligned}
$$

A slightly smaller space (of order of smoothness $s$ ) is the Besov space $B_{p}^{s, 1}, 1<$ $p<\infty$. The norm is given by

$$
\|f\|_{\dot{B}_{p}^{s, 1}}=\sum_{k \in Z} 2^{k s}\left\|f_{k}\right\|_{L^{p}}
$$


This space serves, among other things, as a useful replacement of the spaces $W^{s, p}$ in the cases, when the Sobolev embedding fails. More precisely, we have $\|u\|_{L^{\infty}} \leq C\|u\|_{B_{p}^{d / p, 1}}$. We also note that since $P_{k}$ (and $P_{<k}$ ) is given by a convolution with $2^{k n} \breve{\varphi}\left(2^{k} \cdot\right)$, we have $\left\|P_{k}\right\|_{L^{p} \rightarrow L^{p}} \leq\left\|2^{k n} \check{\varphi}\left(2^{k} \cdot\right)\right\|_{L^{1}} \lesssim 1$ for all $1 \leq p \leq \infty$.

Next, we make several useful observations regarding the Gagliardo-Nirenberg inequalities for such spaces. First, for all $\alpha, \beta>0$ and any $s$, we have

$$
\|f\|_{\dot{B}_{2}^{s, 1}} \leq C_{\alpha, \beta}\|f\|_{\dot{H}^{s+\alpha}}^{\beta /(\alpha+\beta)}\|f\|_{\dot{H}^{s-\beta}}^{\alpha /(\alpha+\beta)} .
$$

Another useful inequality in the same spirit is the following. Assuming $0<a<b$, we have

$$
\|f\|_{\dot{H}^{s+a}}\|f\|_{\dot{H}^{s-a}} \leq C_{a, b}\|f\|_{\dot{H}^{s+b}}\|f\|_{\dot{H}^{s-b}} .
$$

In fact, one can show (2.5) by simply applying (2.4) to each term $\|f\|_{\dot{H}^{s \pm a}} \leq$ $\|f\|_{\dot{B}_{2}^{s \pm a, 1}}$.

\subsection{Littlewood-Paley decompositions of products}

Given the product structure of the nonlinearities in (1.3), we will need to analyze the action of the Littlewood-Paley operators on products. Since supp $\mathcal{F}(f g) \subseteq$ $\operatorname{supp}(\hat{f})+\operatorname{supp}(\hat{g})$, one has

$$
\begin{gathered}
P_{k}(f g)=P_{k}\left(f g_{\geq k+5}\right)+P_{k}\left(f g_{\leq k-5}\right)+P_{k}\left(f g_{k-5<\cdot<k+5}\right) \\
=\sum_{l \geq k+5} P_{k}\left(f_{\sim l} g_{l}\right)+P_{k}\left(f_{k-2 \leq-\leq k+2} g_{\leq k-5}\right) \\
\quad+P_{k}\left(f_{<k+10} g_{k-5<\cdot<k+5}\right) .
\end{gathered}
$$

The first sum gives the so-called "high-high" interactions, whereas the second and the first term give the "high-low" and "low-high" interactions. Usually there is a symmetry ${ }^{1}$ between the $f$ and $g$ in which case, one combines the "high-low" and "low-high" terms.

Similarly for products of three functions, we have the formula

$$
\begin{aligned}
P_{k}(f g h)=P_{k}( & \left.f_{\sim k} g_{<k-10} h_{<k-10}\right)+P_{k}\left(f_{<k-10} g_{\sim k} h_{<k-10}\right) \\
& +P_{k}\left(f_{<k-10} g_{<k-10} h_{\sim k}\right) \\
& +\sum_{l_{1}, l_{2}, l_{3}: \operatorname{med}\left(l_{1}, l_{2}, l_{3}\right) \geq k-10} P_{k}\left(f_{l_{1}} g_{l_{2}} h_{l_{3}}\right),
\end{aligned}
$$

where med $(a, b, c)$ denotes the median of the three numbers.

\footnotetext{
${ }^{1}$ if for example they are the same function or live in the same function space.
} 
We introduce the operator $|\nabla|^{S}$ by

$$
\widehat{|\nabla|^{s} f}:=|\xi|^{s} \hat{f}(\xi) \text {. }
$$

A basic property of such operators, that will be frequently used throughout the paper, is that $\left\|P_{k}|\nabla|^{s} f\right\|_{L^{p}} \sim 2^{k s}\left\|P_{k} f\right\|_{L^{p}}$. The following lemma yields estimates of various commutator terms that appear in the argument. Its proof is classical and can be found for example in [14, Lemma 4.3].

Lemma 2.1. Let $1<q, q_{1}, q_{2}<\infty$ and $1 \leq r_{1}, r_{2}, r \leq \infty$ be given such that $1 / q=1 / q_{1}+1 / q_{2}$ and $1 / r=1 / r_{1}+1 / r_{2}$. Then, for every integer $k$ and for every two Schwartz functions $u, v$,

$$
\left\|P_{k}\left(u v_{k-5<\cdot<k+5}\right)-u v_{k}\right\|_{L^{q} L^{r}} \lesssim 2^{-k}\|\nabla u\|_{L^{q_{1}} L^{r_{1}}}\|v\|_{L^{q_{2} L^{r_{2}}}} .
$$

Another result that will prove useful in the sequel is the fractional derivatives product rule. There are many variants (see for example [1, Proposition 2.1.1]), but we will use the following version.

Lemma 2.2. Let $s>0$ and $u, v$ be functions in the Schwartz class $S$. Then, for every $1<p, r_{1}, r_{2}<\infty$ and $1 \leq q_{1}, q_{2} \leq \infty$ satisfying $1 / p=1 / q_{1}+1 / r_{1}=$ $1 / q_{2}+1 / r_{2}$, one has

$$
\|u v\|_{\dot{W}^{p, s}} \leq C\left(\|u\|_{L^{q_{1}}}\|v\|_{\dot{W}^{r_{1}, s}}+\|u\|_{\dot{W}^{q_{2}, s}}\|v\|_{L^{r_{2}}}\right) .
$$

\subsection{Decay and Strichartz estimates for the wave equation}

Definition 2.3. Let $n \geq 2$. We say that a pair $(q, r)$ is (wave) Strichartz admissible, if $2<q, r<\infty, 1 / q+(n-1) /(2 r) \leq(n-1) / 4$.

For the linear wave equation

$$
\left\{\begin{array}{l}
\square w=F \\
w(x, 0)=f(x), \\
w_{t}(x, 0)=g(x),
\end{array}\right.
$$

we have the following estimates, [7], for any Strichartz admissible pairs $(q, r)$, $(\tilde{q}, \tilde{r})$ and for any real $\alpha$ :

$$
\begin{aligned}
& \left\||\nabla|^{1 / q+n / r+\alpha} w\right\|_{L_{t}^{q} L_{x}^{r}} \\
& \quad \leq C\left(\|f\|_{\dot{H}^{n / 2+\alpha}}+\|g\|_{\dot{H}^{n / 2+\alpha-1}}+\left\||\nabla|^{1 / \tilde{q}^{\prime}+n / \tilde{r}^{\prime}+\alpha-2} F\right\|_{L_{t}^{\tilde{q}^{\prime}} L_{x}^{\tilde{r}^{\prime}}}\right) .
\end{aligned}
$$

Note that the Strichartz estimates above remain valid in the "forbidden" case $(n, q, r)=(3,2, \infty)$, provided the data and/or the forcing term are radial! 
In addition to that, if $F=0$, one has the decay estimate

$$
\sup _{x}|w(t, x)| \leq \frac{C}{t^{(n-1) / 2}}\left[\left\||\nabla|^{n-1} f\right\|_{L^{1}\left(\mathbb{R}^{n}\right)}+\left\||\nabla|^{n-2} g\right\|_{L^{1}\left(\mathbb{R}^{n}\right)}\right] .
$$

If one interpolates between the decay estimate (2.8) and the standard energy estimate $\|w\|_{L^{2}} \leq\|f\|_{L^{2}}+\|g\|_{\dot{H}^{-1}}$, we obtain for all $2 \leq p \leq \infty$,

$$
\begin{aligned}
\|w(t)\|_{L^{p}\left(\mathbb{R}^{n}\right)} \lesssim t^{-(n-1)\left(\frac{1}{2}-\frac{1}{p}\right)}\left[\left\||\nabla|^{(n-1)\left(1-\frac{2}{p}\right)} f\right\|_{L^{p^{\prime}}\left(\mathbb{R}^{n}\right)}\right. & \\
& \left.+\left\||\nabla|^{(n-1)\left(1-\frac{2}{p}\right)-1} g\right\|_{L^{p^{\prime}}\left(\mathbb{R}^{n}\right)}\right] .
\end{aligned}
$$

\section{Energy estimates}

Our next proposition contains the main energy estimate that will allow us to control the growth of the Sobolev norms of the solutions of (1.3). Note that this will remain true as long as the a priori smallness condition (3.1) below holds. Clearly, to close the argument, one will then need an additional estimate to ensure that this quantity stays small, given that the initial data is small in an appropriate sense.

Proposition 3.1. For every $n \geq 1$, there exists a constant $C=C(n)$ and $\delta=\delta(n)$, so that: For every classical solution $\phi$ of (1.3) and for every $T>0$, so that

$$
\begin{aligned}
\left\|\nabla_{t x} \nabla_{x} \phi\right\|_{L^{2}(0, T) L^{\infty}}\left\|\nabla_{t x} \phi\right\|_{L^{2}(0, T) L_{x}^{\infty}} & \\
& +\left\|\nabla_{t x} \phi\right\|_{L_{t x}^{\infty}}^{2}+\left\|\nabla_{t x} \phi\right\|_{L^{\infty}(0, T) \dot{H}^{n / 2}}^{2} \leq \delta^{2},
\end{aligned}
$$

one has

$$
\left(\sum_{k} 2^{2 k s}\left\|\nabla_{x t} \phi_{k}\right\|_{L^{\infty}(0, T) L^{2}}^{2}\right)^{1 / 2} \leq C\left(\|f\|_{\dot{H}^{s+1}}+\|g\|_{\dot{H}^{s}}\right)
$$

for every $s>1$. Moreover,

$$
\left\||\nabla|^{s-1} \partial_{t t} \phi\right\|_{L^{\infty}(0, T) L^{2}} \leq C\left(\|f\|_{\dot{H}^{s+1}}+\|g\|_{\dot{H}^{s}}\right) .
$$

Remark 3.2. (1) Note that in the requirements of Proposition 3.1, we do not require the data $(f, g)$ to be small in any other way but (3.1). Note also, that (3.1) is a scale invariant quantity with respect to the natural scaling $\phi \rightarrow$ $\phi_{\lambda}=\lambda^{-1} \phi(\lambda x, \lambda t)$.

(2) Proposition 3.1 may be viewed as a generalization of the classical energy estimate (see [8], [5], see also (2.11) in [10]) for the solution of the equation (system)

$$
\square w+\sum_{j, k=0}^{n} \gamma^{j k}(t, x) \partial_{j} \partial_{k} w=F .
$$


Namely, assuming $\sum_{j, k=0}^{n}\left|\gamma^{j k}(t, x)\right| \ll 1$, one concludes

$$
\begin{aligned}
\left\|\nabla_{t x} w(t)\right\|_{L^{2}} \lesssim \exp & \left(\int_{0}^{t} c\left|\gamma^{\prime}(\tau)\right| d \tau\right)\left\|\nabla_{t x} w(0)\right\|_{L^{2}} \\
& +\int_{0}^{t} \exp \left(\int_{0}^{s} c\left|\gamma^{\prime}(\tau)\right| d \tau\right)\|F(s, \cdot)\|_{L^{2}} d s .
\end{aligned}
$$

Note that the smallness condition on $\sum_{j, k=0}^{n}\left|\gamma^{j k}(t, x)\right|$, where $\gamma \sim \partial \phi \partial \phi$ (see (3.6) below) is part of our requirement (3.1). Note also that the quantity $\int_{0}^{t}\left|\gamma^{\prime}(\tau)\right| d \tau$ is controlled by $\left\|\nabla_{t x}^{2} \phi\right\|_{L^{2}(0, T) L^{\infty}}\left\|\nabla_{t x} \phi\right\|_{L^{2}(0, T) L_{x}^{\infty}}$, which appears in (3.1).

The idea of the proof is to project the hyperbolic equation (1.3) to LittlewoodPaley pieces and then track their time evolution. As always, the main terms are the ones with the derivatives falling on the entries with the highest frequency. By the structure of the equation, it will turn out that integration by parts (as in the classical arguments) will properly take care of the main terms to derive (3.2) as long as the a priori smallness assumptions (3.1) holds. In fact, our first step will be to show that (3.1) implies, for solutions of (1.3), the related smallness condition

$$
\begin{aligned}
& \left\|\nabla_{t x}^{2} \phi\right\|_{L^{2}(0, T) L}\left\|\nabla_{t x} \phi\right\|_{L^{2}(0, T) L_{x}^{\infty}} \\
& \quad+\left\|\nabla_{t x} \phi\right\|_{L_{t x}^{\infty}}^{2}+\left\|\nabla_{t x} \phi_{k}\right\|_{L^{\infty}(0, T) \dot{H}^{n / 2}}^{2} \leq C \delta^{2}=: \varepsilon^{2},
\end{aligned}
$$

which will be more convenient in the subsequent arguments. Note that the only difference between (3.1) and (3.4) is the presence of the term $\left\|\nabla_{t x}^{2} \phi\right\|_{L^{2}(0, T) L^{\infty}}$ versus $\left\|\nabla_{t x} \nabla_{x} \phi\right\|_{L^{2}(0, T) L^{\infty}}$. Thus, we need to show

$$
\left\|\partial_{t}^{2} \phi\right\|_{L^{2}(0, T) L^{\infty}} \leq C\left\|\nabla_{t x} \nabla_{x} \phi\right\|_{L^{2}(0, T) L^{\infty}}
$$

given (3.1) for classical solutions $\phi$ of (1.3). Indeed, by the form of equation (1.3), we can rewrite it in the following schematic form:

$$
\partial_{t t}^{2} \phi=\Delta \phi+A(\phi)\left[\nabla_{t x} \nabla_{x} \phi\right]+B(\phi)\left[\partial_{t t}^{2} \phi\right],
$$

where $A(\phi), B(\phi) \sim \partial \phi \partial \phi$. Hence, taking $L^{2} L^{\infty}$ norms on both sides of (3.6), we see that

$$
\begin{gathered}
\left\|\partial_{t t}^{2} \phi\right\|_{L^{2}(0, T) L_{x}^{\infty}} \leq\|\Delta \phi\|_{L^{2}(0, T) L_{x}^{\infty}}+\|A(\phi)\|_{L_{t x}^{\infty}}\left\|\partial_{t t}^{2} \phi\right\|_{L^{2}(0, T) L_{x}^{\infty}} \\
+\|B(\phi)\|_{L_{t x}^{\infty}}\left\|\nabla_{t x} \nabla_{x} \phi\right\|_{L^{2}(0, T) L_{x}^{\infty}}
\end{gathered}
$$


Taking into account $\|A(\phi)\|_{L_{t x}^{\infty}}+\|B(\phi)\|_{L_{t x}^{\infty}} \leq C\left\|\nabla_{t x} \phi\right\|_{L_{t x}^{\infty}}^{2} \leq C \delta^{2} \ll 1$, according to (3.1), we get (3.5) and hence (3.4) holds.

We continue now with the proof of the energy estimates in Proposition 3.1 under the smallness assumption (3.4). In order to treat systematically the error terms that arise, we have the following definition.

Definition 3.3. We say that a function $\Psi$ is of type $\operatorname{Err}^{k}$, if for every $T>0$, one has

$$
\|\Psi\|_{L^{1}(0, T) L^{2}} \leq C_{n} 2^{-k}\|\nabla \phi\|_{L^{2} L^{\infty}}\left\|\nabla^{2} \phi\right\|_{L^{2} L^{\infty}} \sum_{l \geq k-5}\left\|\nabla_{x t}^{2} \phi_{l}\right\|_{L_{[0, T]}^{\infty} L_{x}^{2}},
$$

where $C_{n}$ is some suitably chosen and fixed number (to be determined below).

The purpose of this definition is to have a quick notation for all the error terms that appear in the process and which are easily estimable by means of Lemma 2.1.

Next, we apply $P_{k}$ to the both sides of the equation. We will show that $\phi_{k}$ satisfies

$$
\square \phi_{k}-Q\left(\phi_{<k-10}, \phi_{<k-10}\right) \square \phi_{k}=-Q\left(\phi_{<k-10}, Q\left(\phi_{<k-10}, \phi_{k}\right)\right) / 2+\operatorname{Err}^{k} .
$$

Proof of (3.7). Using that $P_{k}$ commutes with constant coefficient differential operators, we get

$$
\square \phi_{k}-P_{k}[Q(\phi, \phi) \square \phi]=-P_{k}[Q(\phi, Q(\phi, \phi))] / 2 .
$$

According to the remarks in Section 2

$$
\begin{aligned}
P_{k} Q(\phi, Q(\phi, \phi))=2 P_{k} & {\left[Q\left(\phi_{<k-10}, Q\left(\phi_{<k-10}, \phi_{k-5<\cdot<k+5}\right)\right)\right] } \\
+ & P_{k}\left[Q\left(\phi_{k-5<\cdot<k+5}, Q\left(\phi_{<k-10}, \phi_{<k-10}\right)\right)\right] \\
& +\sum_{l_{1}, l_{2} l_{3}: \operatorname{med}\left(l_{1}, l_{2}, l_{3}\right) \geq k-10} P_{k}\left[\partial \phi_{l_{1}} \partial \phi_{l_{2}} \partial^{2} \phi_{l_{3}}\right],
\end{aligned}
$$

where in the last term and henceforth, we use $\partial$ to denote the presence of a derivative with respect to any of the variables $t, x_{1}, \ldots, x_{n}$. We will show that the last two terms in (3.8) are of type $\mathrm{Err}^{k}$, whereas for the first term, we have the representation

$$
P_{k} Q\left(\phi_{<k-10}, Q\left(\phi_{k-10}, \phi_{k-5<<<k+5}\right)\right)=Q\left(\phi_{<k-10}, Q\left(\phi_{<k-10}, \phi_{k}\right)\right)+\mathrm{Err}^{k} .
$$


We start treating the corresponding terms systematically. Considering the term $P_{k}\left[Q\left(\phi_{k-5<<k+5}, Q\left(\phi_{<k-10}, \phi_{<k-10}\right)\right)\right]$, we estimate by Hölder's inequality

$$
\begin{aligned}
\| P_{k} Q & \left(\phi_{k-5<\cdot<k+5}, Q\left(\phi_{<k-10}, \phi_{<k-10}\right)\right) \|_{L^{1} L^{2}} \\
& \leq C\left\|\partial \phi_{k-5<\cdot<k+5}\right\|_{L^{\infty} L^{2}}\left\|\partial^{2} \phi\right\|_{L^{2} L^{\infty}}\|\partial \phi\|_{L^{2} L^{\infty}} \\
& \leq C 2^{-k}\left\|\partial^{2} \phi\right\|_{L^{2} L^{\infty}}\|\partial \phi\|_{L^{2} L^{\infty}}\left\|\nabla^{2} \phi_{>k-5}\right\|_{L^{\infty} L^{2}},
\end{aligned}
$$

where in the last inequality, we have used

$$
\left\|\partial \phi_{k-5<\cdot<k+5}\right\|_{L^{\infty} L^{2}} \leq C 2^{-k}\left\|\nabla^{2} \phi_{>k-5}\right\|_{L^{\infty} L^{2}} .
$$

Thus, $P_{k}\left[Q\left(\phi_{k-5<\cdot<k+5}, Q\left(\phi_{<k-10}, \phi_{<k-10}\right)\right)\right]=\operatorname{Err}^{k}$. Note that the constant $C$ depends only on the dimension $n$.

Regarding the last term in (3.8), $\sum_{l_{1}, l_{2} l_{3}: \operatorname{med}\left(l_{1}, l_{2}, l_{3}\right) \geq k-10} P_{k}\left[\partial \phi_{l_{1}} \partial \phi_{l_{2}} \partial^{2} \phi_{l_{3}}\right]$, we observe that by symmetry, we may assume $l_{2} \geq l_{1}$. Also, it is clear that the most difficult case occurs when most derivatives fall on the high-frequency term, that is $l_{3} \geq l_{2}$. So, we restrict ourselves to the case when the summation has the extra restriction $l_{3} \geq l_{2} \geq l_{1}$. We have

$$
\begin{aligned}
\| & \sum_{l_{1} \leq l_{2} \leq l_{3}: l_{2} \geq k-10} P_{k}\left[\partial \phi_{l_{1}} \partial \phi_{l_{2}} \partial^{2} \phi_{l_{3}}\right] \|_{L^{1} L^{2}} \\
\leq & \left\|\sum_{l_{2} \leq l_{3}: l_{2} \geq k-10} \partial \phi_{\leq l_{2}} \partial \phi_{l_{2}} \partial^{2} \phi_{l_{3}}\right\|_{L^{1} L^{2}} \\
& \lesssim\|\nabla \phi\|_{L^{2} L^{\infty}} \sum_{l_{2}, l_{3} \geq k-10}\left\|\partial^{2} \phi_{l_{3}}\right\|_{L^{\infty} L^{2}}\left\|\partial \phi_{l_{2}}\right\|_{L^{2} L^{\infty}} \\
\leq & C 2^{-k}\|\nabla \phi\|_{L_{t}^{2} L_{x}^{\infty}}\left\|\nabla^{2} \phi\right\|_{L_{t}^{2} L_{x}^{\infty}} \sum_{l \geq k-10}\left\|\nabla^{2} \phi_{l}\right\|_{L^{\infty} L^{2}},
\end{aligned}
$$

where in the last step, we have used that $\left\|\partial \phi_{l_{2}}\right\|_{L_{t}^{2} L_{x}^{\infty}} \leq C 2^{-k}\left\|\nabla^{2} \phi\right\|_{L_{t}^{2} L_{x}^{\infty}}$, since $l_{2}>k-10$. Thus, we conclude again

$$
\sum_{l_{1}, l_{2} l_{3}: \operatorname{med}\left(l_{1}, l_{2}, l_{3}\right) \geq k-10} P_{k}\left[\partial \phi_{l_{1}} \partial \phi_{l_{2}} \partial^{2} \phi_{l_{3}}\right]=\operatorname{Err}^{k},
$$

as well. We now turn our attention on (3.9). By Lemma 2.1

$$
\begin{aligned}
\| P_{k}[ & \left.Q\left(\phi_{<k-10}, Q\left(\phi_{k-10}, \phi_{k-5<\cdot<k+5}\right)\right)\right]-Q\left(\phi_{<k-10}, Q\left(\phi_{<k-10}, \phi_{k}\right)\right) \|_{L^{1} L^{2}} \\
& \leq C 2^{-k}\left\|\nabla_{x}\left(\partial \phi_{<k-10} \partial \phi_{k-10}\right)\right\|_{L^{1} L^{\infty}}\left\|\nabla_{x t}^{2} \phi_{k-5<\cdot<k+5}\right\|_{L^{\infty} L^{2}} \\
& \leq C 2^{-k}\|\nabla \phi\|_{L^{2} L^{\infty}}\left\|\nabla^{2} \phi\right\|_{L^{2} L^{\infty}}\left\|\nabla_{x t}^{2} \phi_{k-5<\cdot<k+5}\right\|_{L^{\infty} L^{2}},
\end{aligned}
$$


which establishes (3.9). Similarly, one argues

$$
\begin{aligned}
& P_{k}[Q(\phi, \phi) \square \phi]=P_{k}[\left.Q\left(\phi_{<k-10}, \phi_{<k-10}\right) \square \phi_{k-5<\cdot<k+5}\right] \\
&+2 P_{k}\left[Q\left(\phi_{<k-10}, \phi_{k-5<<k+5}\right) \square \phi_{<k-10}\right] \\
&+\sum_{l_{1}, l_{2}, l_{3}: \operatorname{med}\left(l_{1}, l_{2}, l_{3}\right) \geq k-10} P_{k}\left[\partial \phi_{l_{1}} \partial \phi_{l_{2}} \partial^{2} \phi_{l_{3}}\right] \\
&=Q\left(\phi_{<k-10}, \phi_{<k-10}\right) \square \phi_{k}+\operatorname{Err}^{k}
\end{aligned}
$$

and whence the function $\phi_{k}$ satisfies (3.7).

Having shown (3.7), we will finish the proof of Proposition 3.1 in a relatively standard manner.

\subsection{Proof of Proposition 3.1}

We use the representation formula (3.7) to perform an energy estimate for $\phi_{k}$, which implies (3.2). We use the well-known identity

$$
2 u_{t} \square u=\partial_{t}\left|\nabla_{x t} u\right|^{2}-2 \sum_{1}^{n} \partial_{j}\left(\partial_{t} u \partial_{j} u\right) .
$$

As in the classical argument, one multiplies both sides of (3.7) by $2^{2 k s} \partial_{t} \phi_{k}$ and integrate in the spatial variable $x$. We get

$$
\begin{aligned}
& 2^{2 k s}\left(\int_{\mathbb{R}^{n}}\left(\square \phi_{k}\right)\left(\partial_{t} \phi_{k}\right) d x-\int Q\left(\phi_{<k-10}, \phi_{<k-10}\right) \square \phi_{k} \partial_{t} \phi_{k} d x\right. \\
& \left.\quad+\frac{1}{2} \int Q\left(\phi_{<k-10}, Q\left(\phi_{<k-10}, \phi_{k}\right)\right) \partial_{t} \phi_{k} d x\right)=2^{2 k s} \int \operatorname{Err}^{k}(t, x) \partial_{t} \phi_{k} d x
\end{aligned}
$$

For the first term, we get from (3.10)

$$
\int_{\mathbb{R}^{n}}\left(\square \phi_{k}\right)\left(\partial_{t} \phi_{k}\right) d x=\frac{1}{2} \partial_{t}\left\|\nabla_{x t} \phi_{k}(t)\right\|_{L^{2}}^{2} .
$$

For the next term, (3.10) and integration by parts yield

$$
\begin{aligned}
\int_{\mathbb{R}^{n}} Q\left(\phi_{<k-10}, \phi_{<k-10}\right)\left(\square \phi_{k}\right)\left(\partial_{t} \phi_{k}\right) d x \\
\quad=\int_{\mathbb{R}^{n}} Q\left(\phi_{<k-10}, \phi_{<k-10}\right)\left(\partial_{t}\left|\nabla_{x t} \phi_{k}\right|^{2}-\partial^{j}\left(\partial_{t} \phi_{k} \partial_{j} \phi_{k}\right)\right) d x \\
\quad=\partial_{t} \int Q\left(\phi_{<k-10}, \phi_{<k-10}\right)\left|\nabla_{x t} \phi_{k}\right|^{2} d x+I(t),
\end{aligned}
$$


where

$$
\begin{aligned}
|I(t)| & \leq \int\left|\nabla_{x t} Q\left(\phi_{<k-10}, \phi_{<k-10}\right) \| \nabla_{x t} \phi_{k}\right|^{2} d x \\
& \leq C\left\|\nabla^{2} \phi\right\|_{L^{\infty}}\|\nabla \phi\|_{L^{\infty}}\left\|\nabla_{x t} \phi_{k}\right\|_{L^{2}}^{2} .
\end{aligned}
$$

Denote

$$
\begin{aligned}
& Z_{k}(t)=2^{2 k s}\left\|\nabla_{x t} \phi_{k}(t)\right\|_{L^{2}}^{2} / 2, \\
& Y_{k}(t)=-2^{2 k s} \int Q\left(\phi_{<k-10}, \phi_{<k-10}\right)\left|\nabla_{x t} \phi_{k}(t)\right|^{2} d x .
\end{aligned}
$$

Rewriting the energy equation and incorporating the estimates yields

$$
\begin{aligned}
\partial_{t}\left(Z_{k}(t)+Y_{k}(t)\right) \leq C 2^{2 k s} & \left\|\nabla^{2} \phi(t)\right\|_{L_{x}^{\infty}}\|\nabla \phi(t)\|_{L_{x}^{\infty}}\left\|\nabla_{x t} \phi_{k}(t)\right\|_{L_{x}^{2}}^{2} \\
& +2^{2 k s}\left\|\nabla_{t x} \phi_{k}\right\|_{L^{2}}\left\|\operatorname{Err}^{k}(t)\right\|_{L^{2}} \\
& -\frac{2^{2 k s}}{2} \int Q\left(\phi_{<k-10}, Q\left(\phi_{<k-10}, \phi_{k}\right)\right) \partial_{t} \phi_{k} d x .
\end{aligned}
$$

Introduce $E_{k}(s, T):=2^{2 k s} \sup _{0 \leq t \leq T}\left\|\nabla_{x t} \phi_{k}(t)\right\|_{L^{2}}^{2}$ and integrate the last equation in $[0, T]$. Taking into account

$$
\begin{aligned}
\sup _{0 \leq t \leq T}\left|Y_{k}(t)\right| & \leq C E_{k}(s, T)\|\nabla \phi\|_{L^{\infty}[0, T] L_{x}^{\infty}}^{2} 2^{2 k s}\left\|\nabla_{t x} \phi_{k}\right\|_{L^{2}}\left\|\operatorname{Err}^{k}(t)\right\|_{L^{2}} \\
& \leq \sigma E_{k}(s, T)+C_{\sigma} 2^{2 k s}\left\|\operatorname{Err}^{k}(t)\right\|_{L^{2}}^{2}
\end{aligned}
$$

for any $\sigma>0$, we conclude that by choosing $\sigma \ll 1$

$$
\begin{aligned}
& E_{k}(s, T) \leq E_{k}(s, 0)+C\left(\left\|\nabla^{2} \phi\right\|_{L_{t}^{2}[0, T] L_{x}^{\infty}\|\nabla \phi\|_{L_{t}^{2}[0, T] L^{\infty}}}\right. \\
& \left.+\|\nabla \phi\|_{L_{t}^{\infty}[0, T] L^{\infty}}^{2}\right) E_{k}(s, T)+2^{2 k s}\left\|\operatorname{Err}^{k}(t)\right\|_{L^{1}[0, T] L^{2}}^{2} \\
& +C 2^{2 k s}\left|\int_{0}^{T} \int Q\left(\phi_{<k-10}, Q\left(\phi_{<k-10}, \phi_{k}\right)\right) \partial_{t} \phi_{k} d x d t\right| \text {. }
\end{aligned}
$$

Furthermore, recall that the condition (3.4) guarantees that the term in front of $E_{k}(s, T)$ on the right-hand side is less than $\varepsilon^{2}$ and therefore, if $\varepsilon$ is chosen sufficiently small, one has

$$
\begin{aligned}
E_{k}(s, T) \leq E_{k}(s, 0)+C 2^{2 k s}\left\|\operatorname{Err}^{k}(t)\right\|_{L^{2}}^{2} & \\
& +C 2^{2 k s}\left|\int_{0}^{T} \int Q\left(\phi_{<k-10}, Q\left(\phi_{<k-10}, \phi_{k}\right)\right) \partial_{t} \phi_{k} d x d t\right|
\end{aligned}
$$


We will now state a few lemmas, which will be crucial to finish the argument. In the first two lemmas, we show that the errors are controlled in terms of $E_{k}(s, T)$. However, this cannot be done directly and it has to proceed in two steps. More precisely, define

$$
\tilde{E}_{k}(s, T):=\sup _{0 \leq t \leq T}\left[2^{2 k s}\left\|\nabla_{x t} \phi_{k}(t)\right\|_{L^{2}}^{2}+2^{2 k(s-1)}\left\|\partial_{t}^{2} \phi_{k}(t)\right\|_{L^{2}}^{2}\right],
$$

that is $\tilde{E}_{k}(s, T)=E_{k}(s, T)+\sup _{0 \leq t \leq T} 2^{2 k(s-1)}\left\|\partial_{t}^{2} \phi_{k}(t)\right\|_{L^{2}}^{2}$.

Lemma 3.4. Let $s>1$. Then one has the estimate

$$
\sum_{k=-\infty}^{\infty} 2^{2 k s}\left\|\operatorname{Err}^{k}(t)\right\|_{L^{1}[0, T] L^{2}}^{2} \leq \varepsilon^{2} D_{n} \sum_{k=-\infty}^{\infty} \tilde{E}_{k}(s, T),
$$

where $D_{n}$ depends on the dimension $n$ only.

However, we will show that based on the form of the equation (1.1), we can control $\sum_{k} \tilde{E}_{k}(s, T)$ back in terms of $\sum_{k} E_{k}(s, T)$.

Lemma 3.5. Let $s>1$ and the smallness condition (3.4) holds. Then there exists a constant $C=C_{n}$, so that

$$
\sum_{k=-\infty}^{\infty} \tilde{E}_{k}(s, T) \leq C \sum_{k=-\infty}^{\infty} E_{k}(s, T) .
$$

Since by our assumptions $\left\|\nabla_{t x} \phi\right\|_{\dot{H}^{n / 2}}\left\|\nabla_{t x} \phi\right\|_{L^{\infty}[0, T] L^{\infty}} \leq \varepsilon^{2}$, Lemma 3.4 and Lemma 3.5 imply

$$
\sum_{k=-\infty}^{\infty} 2^{2 k s}\left\|\operatorname{Err}^{k}(t)\right\|_{L^{1}[0, T] L^{2}}^{2} \leq D_{n} \varepsilon^{2} \sum_{k=-\infty}^{\infty} E_{k}(s, T) .
$$

The next lemma concerns the space time integral of the null form.

Lemma 3.6. We have

$$
\begin{gathered}
\left|\int_{0}^{T} \int Q\left(\phi_{<k-10}, Q\left(\phi_{<k-10}, \phi_{k}\right)\right) \partial_{t} \phi_{k} d x d t\right| \\
\leq C\left(\left\|\nabla^{2} \phi(t)\right\|_{L_{x}^{\infty}\|\nabla \phi(t)\|_{L_{x}^{\infty}}\left\|\nabla_{x t} \phi_{k}(t)\right\|_{L_{x}^{2}}^{2}}\right. \\
\left.+\left\|\nabla_{t x} \phi_{k}\right\|_{L^{\infty}[0, T] L_{x}^{2}}^{2}\left\|\nabla_{t x} \phi\right\|_{L_{t x}^{\infty}}^{2}\right) .
\end{gathered}
$$


As a consequence,

$$
\sum_{k} 2^{2 k s}\left|\int_{0}^{T} \int Q\left(\phi_{<k-10}, Q\left(\phi_{<k-10}, \phi_{k}\right)\right) \partial_{t} \phi_{k} d x d t\right| \leq C_{n} \varepsilon^{2} \sum_{k} E_{k}(s, T) .
$$

Lemma 3.4, Lemma 3.5 and Lemma 3.6 are proved in the Appendix. We now assume their validity to finish the proof of Proposition 3.1. Namely, as a consequence of (3.12) and (3.13), we get (after summation in $k$ in (3.11))

$$
\sum_{k=-\infty}^{\infty} E_{k}(s, T) \leq C \varepsilon^{2} \sum_{k=-\infty}^{\infty} E_{k}(s, T)+\sum_{k=-\infty}^{\infty} E_{k}(s, 0),
$$

which shows that again, for $\varepsilon \ll 1$, one has the inequality

$$
\sum_{k=-\infty}^{\infty} E_{k}(s, t) \leq C\|f\|_{\dot{H}^{s+1}}^{2}+C\|g\|_{\dot{H}^{s}}^{2},
$$

which is the statement of (3.2) in Proposition 3.1. Regarding (3.3), we only need to combine the last estimate with Lemma 3.5 to obtain

$$
\begin{aligned}
\left\||\nabla|^{s-1} \partial_{t t} \phi\right\|_{L^{\infty} L^{2}} & \leq\left(\sum_{k=-\infty}^{\infty} \tilde{E}_{k}(s, T)\right)^{1 / 2} \lesssim\left(\sum_{k=-\infty}^{\infty} E_{k}(s, T)\right)^{1 / 2} \\
& \lesssim\|f\|_{\dot{H}^{s+1}}+\|g\|_{\dot{H}^{s}}
\end{aligned}
$$

\section{Global regularity for the minimal surface equation}

In this section, we present the proof of Theorem 1.1. Assume that the initial data $(f, g)$ is $C^{\infty}$ smooth (in addition to the conditions required in Theorem 1.1). A classical short term existence theorem applies and we have a local smooth solution up to at least some positive time. The idea then is to show that the energy estimates in Proposition 3.1 persist indefinitely.

More precisely, we start the argument with the claim that the smallness condition (1.4) implies the smallness condition (3.1) in some (initially small) interval $\left[0, T_{0}\right]$ in Proposition 3.1, whence we will have the energy estimates (3.2) for any $s>1$. Indeed, the first term in (3.1), $\left\|\nabla_{t x} \nabla_{x} \phi\right\|_{L^{2}[0, T] L^{\infty}}\left\|\nabla_{t x} \phi\right\|_{L^{2}[0, T] L^{\infty}}$, can be made small just by taking $T$ to be small enough. For the other two terms, we use the Bernstein estimates to bound

$$
\left\|\nabla_{t x} \phi\right\|_{L_{t x}^{\infty}}^{2}+\left\|\nabla_{t x} \phi_{k}\right\|_{L^{\infty}(0, T) \dot{H}^{n / 2}}^{2} \leq C\left\|\nabla_{t x} \phi\right\|_{L_{t}^{\infty} \dot{B}_{2}^{n / 2,1}}^{2},
$$


which again is small by (1.4), the Gagliardo-Nirenberg inequality (2.4) and a standard continuity argument in some (maybe even smaller than before) non-trivial time interval $\left[0, \tilde{T}_{0}\right]$. Summarizing, we have ensured that in some non-trivial time interval $\left[0, \tilde{T}_{0}\right]$, the smallness condition (3.1) in Proposition 3.1 holds. Denote

$$
\begin{array}{r}
T^{*}=\sup \left\{T>0:\left\|\nabla_{t x} \nabla_{x} \phi\right\|_{L_{T}^{2} L^{\infty}}\left\|\nabla_{t x} \phi\right\|_{L_{T}^{2} L^{\infty}}+\left\|\nabla_{t x} \phi\right\|_{L_{t x}^{\infty}}^{2}\right. \\
\left.+\left\|\nabla_{t x} \phi_{k}\right\|_{L_{T}^{\infty} \dot{H}^{n / 2}}^{2} \leq \delta^{2}\right\} .
\end{array}
$$

If we show that $T^{*}=\infty$, then we can clearly apply the energy estimates in Proposition 3.1 for all times and we are done. In particular, we would obtain the estimate for $\|\phi\|_{L_{t}^{\infty} \dot{H}^{s}}$ stated in (1.5).

Thus, assume $0<T<T^{*}$. It follows that the smallness condition (3.1) is satisfied and by Proposition 3.1, for all $s>1$,

$$
\|\phi\|_{L^{\infty}(0, \infty) \dot{H}^{s}} \leq C\|(f, g)\|_{\dot{H}^{s} \times \dot{H}^{s-1}} .
$$

By Bernstein's inequality, (2.4) with $\alpha=\beta=\sigma \in(0,1 / 2)$ and (3.2) with $s=$ $n / 2 \pm \sigma$,

$$
\begin{aligned}
\| \nabla_{t x} \phi & \left\|_{L_{t x}^{\infty}}^{2}+\right\| \nabla_{t x} \phi_{k} \|_{L^{\infty}(0, T) \dot{H}^{n / 2}}^{2} \\
& \leq C\left\|\nabla_{t x} \phi\right\|_{L_{t}^{\infty} \dot{B}_{2}^{n / 2,1}}^{2} \\
& \leq C_{\sigma}\left\|\nabla_{t x} \phi\right\|_{L_{t}^{\infty} \dot{H}^{n / 2+\sigma}}\left\|\nabla_{t x} \phi\right\|_{L_{t}^{\infty} \dot{H}^{n / 2-\sigma}} \\
& \leq C_{\sigma}\left(\|f\|_{\dot{H}^{n / 2+\sigma+1}}+\|g\|_{\dot{H}^{n / 2+\sigma}}\right)\left(\|f\|_{\dot{H}^{n / 2-\sigma+1}}+\|g\|_{\dot{H}^{n / 2-\sigma}}\right) .
\end{aligned}
$$

To estimate the last expression, we apply (2.5). We have lots of options there, but one obtains, for example, the bound

$$
\begin{aligned}
& \|(f, g)\|_{\dot{H}^{n / 2+\sigma+1} \times \dot{H}^{n / 2+\sigma}}\|(f, g)\|_{\dot{H}^{n / 2-\sigma+1} \times \dot{H}^{n / 2-\sigma}} \\
& \quad \leq C\|(f, g)\|_{\dot{H}^{n / 2+5 / 2} \times \dot{H}^{n / 2+3 / 2}}\|(f, g)\|_{\dot{H}^{n / 2-1 / 2} \times \dot{H}^{n / 2-3 / 2}}<C \kappa^{2},
\end{aligned}
$$

according to the smallness condition (1.4) in Theorem 1.1. Thus, the last two terms in the definition of $T^{*}$ stay small uniformly in $T \rightarrow T^{*}$. In particular, a choice of $\kappa$ such that $C \kappa^{2} \ll \delta^{2}$ will ensure that these quantities stay away from $\delta^{2}$, even as $T \rightarrow T^{*}$.

It remains to show that the same goes for $\left\|\nabla_{t x} \nabla_{x} \phi\right\|_{L_{T}^{2} L^{\infty}}\left\|\nabla_{t x} \phi\right\|_{L_{T}^{2} L^{\infty}}$. This is achieved via the Strichartz estimates, which will tie back both $\left\|\nabla_{t x} \nabla_{x} \phi\right\|_{L_{T}^{2} L^{\infty}}$ and $\left\|\nabla_{t x} \phi\right\|_{L_{T}^{2} L^{\infty}}$ to some Sobolev norms of the solution $\phi$. It is at this stage that we will have to consider the case $n \geq 4$ (or $n=3$ with radial data) separately from the case $n=3$. 


\subsection{The case $n \geq 4$}

First, let us mention that even for $n \geq 4,(2, \infty)$ is not an admissible Strichartz pair in the strict sense of Definition 2.3. However, we can apply Sobolev embedding and reduce the estimates for $\left\|\nabla_{t x} \phi\right\|_{L_{T}^{2} L^{\infty}}$ and $\left\|\nabla_{t x} \nabla_{x} \phi\right\|_{L_{T}^{2} L^{\infty}}$ to a Strichartz admissible pair. Starting with $\left\|\nabla_{t x} \phi\right\|_{L_{T}^{2} L^{\infty}}$, we have

$$
\left\|\nabla_{t x} \phi\right\|_{L_{T}^{2} L^{\infty}} \leq C\left\|\nabla_{t x} \phi\right\|_{L_{T}^{2} \dot{B}_{2(n-1) /(n-3)}^{n(n-3) / 2(n-1), 1}} .
$$

The drawback of this last step, even though it reduces to the case of Strichartz admissible pair, is that the $x$ dependence is measured in the slightly more restrictive Besov norms. By Gagliardo-Nirenberg's inequality however,

$$
\begin{aligned}
& \left\|\nabla_{t x} \phi\right\|_{L_{T}^{2} \dot{B}_{2(n-1) /(n-3)}^{n(n-3) / 2(n-1), 1}} \\
& \quad \leq B_{\mu}\left\|\nabla_{t x} \phi\right\|_{L_{T}^{2} \dot{W}}^{1 / 2} \frac{2(n-1), \frac{n(n-3)}{n-3}, \frac{2(n-1)}{n-\mu}}{\left\|\nabla_{t x} \phi\right\|^{1 / 2}}{ }_{L_{T}^{2} \dot{W}}^{\frac{2(n-1)}{n-3}, \frac{n(n-3)}{2(n-1)}-\mu} .
\end{aligned}
$$

Recall (see (3.6))

$$
\square \phi=A(\phi)\left[\nabla_{t x} \nabla_{x} \phi\right]+B(\phi)\left[\partial_{t t}^{2} \phi\right],
$$

where $A(\phi), B(\phi) \sim \partial \phi \partial \phi$. The Strichartz estimates (2.7), applied for $(q, r)=$ $\left(2, \frac{2(n-1)}{n-3}\right)$, and Lemma 2.2 yield

$$
\begin{aligned}
& \left\|\nabla_{t x} \phi\right\|_{L_{T}^{2} \dot{W}} \frac{2(n-1)}{n-3}, \frac{n(n-3)}{2(n-1)} \pm \mu \\
& \leq C\|(f, g)\|_{\dot{H}^{n / 2+1 / 2 \pm \mu} \times \dot{H}^{n / 2-1 / 2 \pm \mu}} \\
& +C\left\||\nabla|^{n / 2-1 / 2 \pm \mu}\left[(\partial \phi \partial \phi) \nabla_{t x} \nabla_{x} \phi+(\partial \phi \partial \phi) \partial_{t t}^{2} \phi\right]\right\|_{L_{T}^{1} L^{2}} \\
& \lesssim\|(f, g)\|_{\dot{H}^{n / 2+1 / 2 \pm \mu} \times \dot{H}^{n / 2-1 / 2 \pm \mu}} \\
& +\|\nabla \phi\|_{L_{T}^{2} L_{x}^{\infty}}^{2}\left\||\nabla|^{n / 2+1 / 2 \pm \mu} \nabla_{t x} \phi\right\|_{L_{T}^{\infty} L^{2}} \\
& +\|\nabla \phi\|_{L_{T}^{2} L_{x}^{\infty}}\left\|\nabla_{t x} \nabla \phi\right\|_{L_{T}^{2} L_{x}^{\infty}}\left\||\nabla|^{n / 2-1 / 2 \pm \mu} \nabla_{t x} \phi\right\|_{L_{T}^{\infty} L^{2}} \\
& +\|\nabla \phi\|_{L_{T}^{2} L_{t x}^{\infty}}^{2}\left\||\nabla|^{n / 2-1 / 2 \pm \mu} \partial_{t t} \phi\right\|_{L_{T}^{\infty} L^{2}}
\end{aligned}
$$

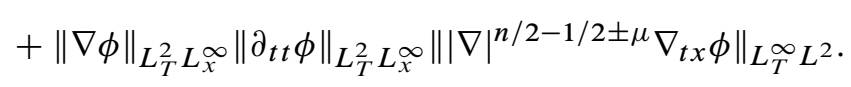


By (3.2), (3.3), (3.5) and taking into account $\left\|\nabla_{t x} \nabla_{x} \phi\right\|_{L_{T}^{2} L^{\infty}}\left\|\nabla_{t x} \phi\right\|_{L_{T}^{2} L^{\infty}} \leq \delta^{2}$ (since $T<T^{*}$ ), we continue our estimation

$$
\begin{aligned}
& \left\|\nabla_{t x} \phi\right\|_{L_{T}^{2} \dot{W}^{\frac{2(n-1)}{n-3}, \frac{n(n-3)}{2(n-1)} \pm \mu}} \\
& \leq C\|(f, g)\|_{\dot{H}^{n / 2+1 / 2 \pm \mu} \times \dot{H}^{n / 2-1 / 2 \pm \mu}} \\
& \quad+C\|\nabla \phi\|_{L_{T}^{2} L_{t x}^{\infty}}^{\infty}\|(f, g)\|_{\dot{H}^{n / 2+3 / 2 \pm \mu} \times \dot{H}^{n / 2+1 / 2 \pm \mu}} .
\end{aligned}
$$

This implies for all $0<T<T^{*}$,

$$
\begin{aligned}
& \left\|\nabla_{t x} \phi\right\|_{L_{T}^{2} \dot{W}} \frac{2(n-1)}{n-3}, \frac{n(n-3)}{2(n-1)} \pm \mu \\
& \leq C_{1}\|(f, g)\|_{\dot{H}^{n / 2+1 / 2 \pm \mu} \times \dot{H}^{n / 2-1 / 2 \pm \mu}} \\
& \quad+C\left\|\nabla_{t x} \phi\right\|_{L_{T}^{2} L_{t x}^{\infty}}^{2}\|(f, g)\|_{\dot{H}^{n / 2+3 / 2 \pm \mu} \times \dot{H}^{n / 2+1 / 2 \pm \mu}} .
\end{aligned}
$$

Now we run a separate continuity argument, which will establish

$$
\left\|\nabla_{t x} \phi\right\|_{L_{T}^{2} \dot{W}^{\frac{2(n-1)}{n-3}, \frac{n(n-3)}{2(n-1)} \pm \mu}} \leq 2 C_{1}\|(f, g)\|_{\dot{H}^{n / 2+1 / 2 \pm \mu} \times \dot{H}^{n / 2-1 / 2 \pm \mu}}
$$

for all $0<T<T^{*}$. We do this as follows. Define

$$
\begin{aligned}
T_{1}=\sup \left\{T \in\left(0, T^{*}\right):\left\|\nabla_{t x} \phi\right\|_{L_{T}^{2} \dot{W}^{\frac{2(n-1)}{n-3}, \frac{n(n-3)}{2(n-1)} \pm \mu}}\right. \\
\quad \leq 2 C_{1}\|(f, g)\|_{\left.\dot{H}^{n / 2+1 / 2 \pm \mu} \times \dot{H}^{n / 2-1 / 2 \pm \mu}\right\}} .
\end{aligned}
$$

For all $0<T<T_{1}$ and by (4.1), we have the following estimate:

$$
\begin{aligned}
& \left\|\nabla_{t x} \phi\right\|_{L_{T}^{2} L_{x}^{\infty}} \\
& \quad \leq 2 B_{\mu} C_{1}\|(f, g)\|_{\dot{H}^{n / 2+1 / 2+\mu} \times \dot{H}^{n / 2-1 / 2+\mu}}^{1 / 2}\|(f, g)\|_{\dot{H}^{n / 2+1 / 2-\mu_{x}} \dot{H}^{n / 2-1 / 2-\mu}}^{1 / 2},
\end{aligned}
$$

which we feed in the right-hand side of (4.2). We obtain

$$
\begin{aligned}
\left\|\nabla_{t x} \phi\right\|_{L_{T}^{2} \dot{W}^{\frac{2(n-1)}{n-3}, \frac{n(n-3)}{2(n-1)} \pm \mu} \leq} \leq C_{1} \| & (f, g) \|_{\dot{H}^{n / 2+1 / 2 \pm \mu} \times \dot{H}^{n / 2-1 / 2 \pm \mu}} \\
+ & 4 B_{\mu}^{2} C_{1}^{2} C\|(f, g)\|_{\dot{H}^{n / 2+1 / 2+\mu} \times \dot{H}^{n / 2-1 / 2+\mu}} \\
& \times\|(f, g)\|_{\dot{H}^{n / 2+1 / 2-\mu} \times \dot{H}^{n / 2-1 / 2-\mu}} \\
& \times\|(f, g)\|_{\dot{H}^{n / 2+3 / 2 \pm \mu} \times \dot{H}^{n / 2+1 / 2 \pm \mu}}
\end{aligned}
$$


An application of (2.5) and (1.4) yields

$$
\begin{aligned}
& \|(f, g)\|_{\dot{H}^{n / 2+1 / 2+\mu} \times \dot{H}^{n / 2-1 / 2+\mu}}\|(f, g)\|_{\dot{H}^{n / 2+1 / 2-\mu} \times \dot{H}^{n / 2-1 / 2-\mu}}
\end{aligned}
$$

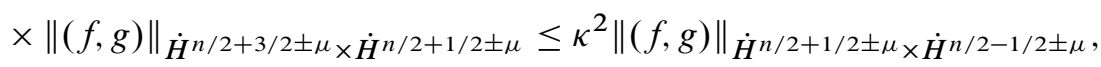

which implies that if $T \in\left(0, T_{1}\right)$, we have the estimate

$$
\begin{aligned}
\left\|\nabla_{t x} \phi\right\| & L_{T}^{2} \dot{W}^{\frac{2(n-1)}{n-3}, \frac{n(n-3)}{2(n-1)} \pm \mu} \\
& \leq\left(C_{1}+C_{2}(\mu, n) \kappa^{2}\right)\|(f, g)\|_{\dot{H}^{n / 2+1 / 2 \pm \mu} \times \dot{H}^{n / 2-1 / 2 \pm \mu}} .
\end{aligned}
$$

Thus, if $\kappa$ satisfies $C_{2} \kappa^{2}<C_{1} / 2$, then we obtain for all $0<T<T_{1}$,

$$
\left\|\nabla_{t x} \phi\right\|_{L_{T}^{2} \dot{W}^{\frac{2(n-1)}{n-3}, \frac{n(n-3)}{2(n-1)} \pm \mu}} \leq \frac{3 C_{1}}{2}\|(f, g)\|_{\dot{H}^{n / 2+1 / 2 \pm \mu} \times \dot{H}^{n / 2-1 / 2 \pm \mu}},
$$

which implies that $T_{1}=T^{*}$. That is

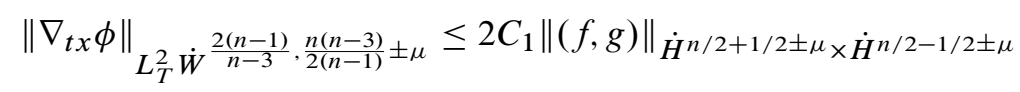

for all $0<T<T^{*}$ and for some constant $C_{1}$ depending only on the dimension. Hence

$$
\begin{aligned}
\left\|\nabla_{t x} \phi\right\|_{L_{T}^{2} L_{x}^{\infty} \leq 2 C_{1}}\|(f, g)\|_{\dot{H}^{n / 2+1 / 2+\mu} \times \dot{H}^{n / 2-1 / 2+\mu}}^{1 / 2} \\
\times\|(f, g)\|_{\dot{H}^{n / 2+1 / 2-\mu} \times \dot{H}^{n / 2-1 / 2-\mu}}^{1 / 2} .
\end{aligned}
$$

The considerations for $\left\|\nabla_{t x} \nabla_{x} \phi\right\|_{L_{T}^{2} L^{\infty}}$ are similar in nature. A GagliardoNirenberg estimate, similar to (4.1), yields control of $\left\|\nabla_{t x} \nabla_{x} \phi\right\|_{L_{T}^{2} L^{\infty}}$ in terms of Strichartz type norms of the solution, which we control below. We have, by the Strichartz estimates and Lemma 2.2,

$$
\begin{aligned}
& \left\|\nabla_{t x} \nabla_{x} \phi\right\|_{L_{T}^{2} \dot{W}} \frac{2(n-1)}{n-3}, \frac{n(n-3)}{2(n-1)} \pm \mu \\
& \lesssim\|(f, g)\|_{\dot{H}^{n / 2+3 / 2 \pm \mu} \times \dot{H}^{n / 2+1 / 2 \pm \mu}} \\
& +\|\nabla \phi\|_{L_{T}^{2} L_{x}^{\infty}}^{2}\left\||\nabla|^{n / 2+3 / 2 \pm \mu} \nabla_{t x} \phi\right\|_{L_{T}^{\infty} L^{2}}
\end{aligned}
$$

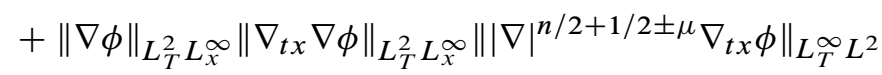

$$
\begin{aligned}
& +\|\nabla \phi\|_{L_{T}^{2} L_{t x}^{\infty}}^{2}\left\||\nabla|^{n / 2+1 / 2 \pm \mu} \partial_{t t} \phi\right\|_{L_{T}^{\infty} L^{2}}
\end{aligned}
$$

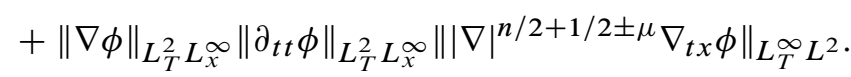


By (3.2), (3.3), (3.5), (4.3),

$$
\begin{aligned}
& \left\|\nabla_{t x} \nabla_{x} \phi\right\|_{L_{T}^{2} \dot{W}^{\frac{2(n-1)}{n-3}, \frac{n(n-3)}{2(n-1)} \pm \mu}} \\
& \leq C\|(f, g)\|_{\dot{H}^{n / 2+3 / 2 \pm \mu} \times \dot{H}^{n / 2+1 / 2 \pm \mu}} \\
& +C\|(f, g)\|_{\dot{H}^{n / 2+1 / 2+\mu} \times \dot{H}^{n / 2-1 / 2+\mu}} \\
& \quad \times\|(f, g)\|_{\dot{H}^{n / 2+1 / 2-\mu} \times \dot{H}^{n / 2-1 / 2-\mu}} \\
& \quad \times\|(f, g)\|_{\dot{H}^{n / 2+5 / 2 \pm \mu} \times \dot{H}^{n / 2+3 / 2 \pm \mu}} \\
& +C\|(f, g)\|_{\dot{H}^{n / 2+1 / 2+\mu} \times \dot{H}^{n / 2-1 / 2+\mu}}^{1 / 2} \\
& \quad \times\|(f, g)\|_{\dot{H}^{n / 2+1 / 2-\mu} \times \dot{H}^{n / 2-1 / 2-\mu}}^{1 / 2} \\
& \quad \times\|(f, g)\|_{\dot{H}^{n / 2+3 / 2 \pm \mu} \times \dot{H}^{n / 2+1 / 2 \pm \mu}}\left\|\nabla_{t x} \nabla_{x} \phi\right\|_{L_{T}^{2} L^{\infty}}
\end{aligned}
$$

Again, due to (2.5) and (1.4)

$\|(f, g)\|_{\dot{H}^{n / 2+1 / 2+\mu} \times \dot{H}^{n / 2-1 / 2+\mu}}\|(f, g)\|_{\dot{H}^{n / 2+1 / 2-\mu} \times \dot{H}^{n / 2-1 / 2-\mu}}$ $\times\|(f, g)\|_{\dot{H}^{n / 2+5 / 2 \pm \mu} \times \dot{H}^{n / 2+3 / 2 \pm \mu}} \leq \kappa^{2}\|(f, g)\|_{\dot{H}^{n / 2+3 / 2 \pm \mu} \times \dot{H}^{n / 2+1 / 2 \pm \mu}}$.

Combining all the estimates that we have for $\left\|\nabla_{t x} \nabla_{x} \phi\right\|_{L_{T}^{2} \dot{W}^{\frac{2(n-1)}{n-3}, \frac{n(n-3)}{2(n-1)} \pm \mu}}$ with the Gagliardo-Nirenberg inequality yields for every $0<T<T^{*}$,

$$
\begin{aligned}
& \left\|\nabla_{t x} \nabla_{x} \phi\right\|_{L_{T}^{2} L \infty}^{2}
\end{aligned}
$$

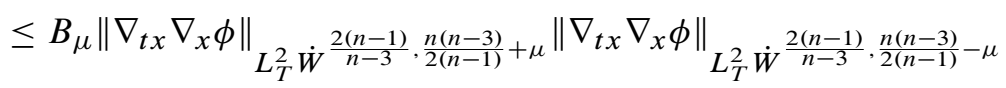

$$
\begin{aligned}
& \leq C B_{\mu}\left(\|(f, g)\|_{\dot{H}^{n / 2+3 / 2+\mu} \times \dot{H}^{n / 2+1 / 2+\mu}}\right. \\
& +\|(f, g)\|_{\dot{H}^{n / 2+1 / 2+\mu} \times \dot{H}^{n / 2-1 / 2+\mu}}^{1 / 2}\|(f, g)\|_{\dot{H}^{n / 2+1 / 2-\mu} \times \dot{H}^{n / 2-1 / 2-\mu}}^{1 / 2} \\
& \left.\times\|(f, g)\|_{\dot{H}^{n / 2+3 / 2+\mu} \times \dot{H}^{n / 2+1 / 2+\mu}}\left\|\nabla_{t x} \nabla_{x} \phi\right\|_{L_{T}^{2} L^{\infty}}\right) \\
& \times\left(\|(f, g)\|_{\dot{H}^{n / 2+3 / 2-\mu} \times \dot{H}^{n / 2+1 / 2-\mu}}\right. \\
& +\|(f, g)\|_{\dot{H}^{n / 2+1 / 2+\mu} \times \dot{H}^{n / 2-1 / 2+\mu}}^{1 / 2}\|(f, g)\|_{\dot{H}^{n / 2+1 / 2-\mu} \times \dot{H}^{n / 2-1 / 2-\mu}}^{1 / 2}
\end{aligned}
$$

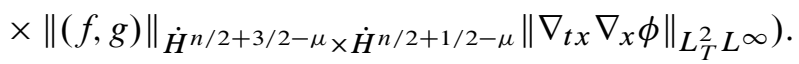


Taking into account various product terms that are less than $\kappa^{2}$ (similar as the argument before), we arrive at

$$
\begin{aligned}
& \left\|\nabla_{t x} \nabla_{x} \phi\right\|_{L_{T}^{2} L^{\infty}}^{2} \\
& \leq C B_{\mu}\left(\|(f, g)\|_{\dot{H}^{n / 2+3 / 2+\mu} \times \dot{H}^{n / 2+1 / 2+\mu}}\|(f, g)\|_{\dot{H}^{n / 2+3 / 2-\mu} \times \dot{H}^{n / 2+1 / 2-\mu}}\right. \\
& \quad+\kappa^{2}\|(f, g)\|_{\dot{H}^{n / 2+3 / 2+\mu} \times \dot{H}^{n / 2+1 / 2+\mu}}^{1 / 2} \\
& \quad \times\|(f, g)\|_{\dot{H}^{n / 2+3 / 2-\mu} \times \dot{H}^{n / 2+1 / 2-\mu}}^{1 / 2}\left\|\nabla_{t x} \nabla_{x} \phi\right\|_{L_{T}^{2} L^{\infty}} \\
& \left.+\kappa^{4}\left\|\nabla_{t x} \nabla_{x} \phi\right\|_{L_{T}^{2} L^{\infty}}^{2}\right),
\end{aligned}
$$

whence for $\kappa$ satisfying $C B_{\mu} \kappa^{4} \leq 1 / 10$, we obtain

$$
\begin{aligned}
\left\|\nabla_{t x} \nabla_{x} \phi\right\|_{L_{T}^{2} L^{\infty}} \leq C_{2} \| & (f, g) \|_{\dot{H}^{n / 2+3 / 2+\mu} \times \dot{H}^{n / 2+1 / 2+\mu}}^{1 / 2} \\
& \times\|(f, g)\|_{\dot{H}^{n / 2+3 / 2-\mu} \times \dot{H}^{n / 2+1 / 2-\mu}}^{1 / 2}
\end{aligned}
$$

for a constant $C_{2}=C_{2}(n)$ and for all $0<T<T^{*}$.

Combining the estimates (4.3) and (4.4) yields

$$
\begin{aligned}
\left\|\nabla_{t x} \phi\right\|_{L_{T}^{2} L^{\infty}}\left\|\nabla_{t x} \nabla_{x} \phi\right\|_{L_{T}^{2} L^{\infty}} & \\
\leq & C_{3}\|(f, g)\|_{\dot{H}^{n / 2+1 / 2+\mu} \times \dot{H}^{n / 2-1 / 2+\mu}}^{1 / 2}\|(f, g)\|_{\dot{H}^{n / 2+1 / 2-\mu} \times \dot{H}^{n / 2-1 / 2-\mu}}^{1 / 2} \\
\quad & \|(f, g)\|_{\dot{H}^{n / 2+3 / 2+\mu} \times \dot{H}^{n / 2+1 / 2+\mu}}^{1 / 2}\|(f, g)\|_{\dot{H}^{n / 2+3 / 2-\mu} \times \dot{H}^{n / 2+1 / 2-\mu}}^{1 / 2} .
\end{aligned}
$$

Since again by (2.5) the last expression is bounded by $C_{3} \kappa^{2}$, it follows that for a choice of $\kappa$, namely $C_{3} \kappa^{2}<\delta^{2} / 10$, where $\delta=\delta_{n}$ is the number from Proposition 3.1, we obtain

$$
\left\|\nabla_{t x} \phi\right\|_{L_{T}^{2} L^{\infty}}\left\|\nabla_{t x} \nabla_{x} \phi\right\|_{L_{T}^{2} L^{\infty}} \leq \delta^{2} / 10 .
$$

Clearly, this implies that all three quantities involved in the definition of $T^{*}$ remain $\ll \delta^{2}$, uniformly in $T$ with $0<T<T^{*}$, as long as $\kappa$ is chosen to be suitably small (depending on $n, \mu$ ). Hence $T^{*}=\infty$ and Theorem 1.1 is proved.

\subsection{The case $n=3$ with radial data}

This case is technically simpler than the case $n \geq 4$. We simply need to observe that even though, formally according to Definition $2.3,(q, r)=(2, \infty)$ is not a Strichartz pair, the Strichartz estimates hold, when the data is radial. Therefore, 
we do not need the intermediary role provided by the Besov spaces, as we needed to do for the case $n \geq 4$. In essence, one can rigorously repeat the argument presented in the previous section with $n=3$ and $\mu=0$, in order to control the required quantity $\left\|\nabla_{t x} \phi\right\|_{L_{T}^{2} L^{\infty}}\left\|\nabla_{t x} \nabla_{x} \phi\right\|_{L_{T}^{2} L^{\infty}}$. We omit the details.

\subsection{The case $n=3$ with non-radial data}

The estimates in this case are more delicate, due to the failure of the endpoint Strichartz estimate at $q=2, r=\infty$. We will instead rely on the following inhomogeneous estimate for the wave equation in $3 \mathrm{D}^{2}$.

Lemma 4.1. Let $w$ satisfy the wave equation $\square w=F, w(x, 0)=0, w_{t}(x, 0)=0$ in $(x, t) \in \mathbb{R}^{3+1}$. Then, for $4<p<\infty$, q satisfying $1 / 2+2 / p=1 / q$ and for every real $\alpha$, there is the estimate

$$
\left\||\nabla|^{\alpha} w\right\|_{L^{2} L^{p}\left(\mathbb{R}^{3}\right)} \leq\left(\sum_{k} 2^{2 k \alpha}\left\|w_{k}\right\|_{L^{2} L_{x}^{p}}^{2}\right)^{1 / 2} \leq C_{p}\left\||\nabla|^{\alpha+1-4 / p} F\right\|_{L_{t}^{q} L^{p^{\prime}}} .
$$

More generally, for every $4<\tilde{p} \leq \infty$, we have the estimates

$$
\left\||\nabla|^{\beta} w\right\|_{L^{2} L^{\tilde{p}}\left(\mathbb{R}^{3}\right)} \leq C_{p}\left\||\nabla|^{\alpha+1-4 / p} F\right\|_{L_{t}^{q} L^{p^{\prime}}}
$$

for any $4<p<\tilde{p}$ and $\alpha=\beta+3(1 / p-1 / \tilde{p})$.

We present the (standard) proof of Lemma 4.1 in the Appendix, but we will show how it implies Theorem 1.3. Recall that we still need to control $\left\|\nabla^{j} \phi\right\|_{L_{T}^{2} L_{X}^{\infty}}$, $j=1,2$. For the fixed $p>4$, we embed this quantities in the following family of norms:

$$
X_{T}(\phi)=\left(\sum_{k} 2^{2 k \alpha}\left\|\phi_{k}\right\|_{L_{T}^{2} L^{p}}^{2}\right)^{1 / 2}
$$

where $0<\alpha \leq 2+3 / p$. By Sobolev embedding, $\left\|\nabla^{j} \phi\right\|_{L_{T}^{2} L_{x}^{\infty}} \leq X_{T}(\phi), j=$ 1,2. Keeping in mind that we control $\sup _{0 \leq t \leq T}\left\||\nabla|{ }^{s} \nabla_{t x} \phi\right\|_{L^{2}} \leq C\left(\|f\|_{H^{s+1}}+\right.$ $\|g\|_{H^{s}}$ ), we proceed with the estimate for $X_{T}(\phi)$. By the form of equation (3.6), we have

$\phi=\cos (t \sqrt{-\Delta}) f+\frac{\sin (t \sqrt{-\Delta})}{\sqrt{-\Delta}} g+\int_{0}^{t} \frac{\sin ((t-s) \sqrt{-\Delta})}{\sqrt{-\Delta}}\left[\partial \phi \partial \phi \partial^{2} \phi\right](s, \cdot) d s$,

2 although nothing is really special in $3 \mathrm{D}$ for such an estimate, but this is what we will need in the proof 
whence, by Lemma 4.1,

$$
\begin{gathered}
X_{T}(\phi) \leq C\left\|\cos (t \sqrt{-\Delta})|\nabla|^{\alpha} f\right\|_{L_{T}^{2} L^{p}}+C\left\|\sin (t \sqrt{-\Delta})|\nabla|^{\alpha-1} g\right\|_{L_{T}^{2} L^{p}} \\
+C\left\||\nabla|^{\alpha+1-4 / p}\left[\partial \phi \partial \phi \partial^{2} \phi\right]\right\|_{L_{T}^{q} L^{p^{\prime}}} .
\end{gathered}
$$

For the terms, arising out of the initial data, we apply the decay estimates (2.9) for the free wave equation, when $t \geq 1$, and the Sobolev embedding $\|h\|_{L^{p}} \lesssim$ $\|h\|_{\dot{H}^{3(1 / 2-1 / p)}}$ and the energy estimates, when $0 \leq t<1$. We get

$$
\begin{aligned}
& \left\|\cos (t \sqrt{-\Delta})|\nabla|^{\alpha} f\right\|_{L_{T}^{2} L^{p}}+\left\|\sin (t \sqrt{-\Delta})|\nabla|^{\alpha-1} g\right\|_{L_{t}^{2} L^{p}} \\
& \leq C\left(\left\||\nabla|^{\alpha+2-4 / p} f\right\|_{L^{p^{\prime}}}+\left\||\nabla|^{\alpha+1-4 / p} g\right\|_{L^{p^{\prime}}}\right)\left(\int_{1}^{T} t^{-2(1-2 / p)} d t\right)^{1 / 2} \\
& \quad+C\left(\left\||\nabla|^{\alpha+3(1 / 2-1 / p)} f\right\|_{L^{2}}+\left\||\nabla|^{\alpha-1+3(1 / 2-1 / p)} g\right\|_{L^{2}}\right) \\
& \leq C\|(f, g)\|_{W^{4-1 / p, p^{\prime}} \times W^{3-1 / p, p^{\prime}}}+\|(f, g)\|_{H^{7 / 2} \times H^{5 / 2}},
\end{aligned}
$$

where in the last inequality, we have used $\alpha \leq 2+3 / p$.

For the term $\left\||\nabla|^{\alpha+1-4 / p}\left[\partial \phi \partial \phi \partial^{2} \phi\right]\right\|_{L_{t}^{q} L^{p^{\prime}}}$, we estimate by the fractional differentiation rule

$$
\begin{aligned}
& \left\||\nabla|^{\alpha+1-4 / p}\left[\partial \phi \partial \phi \partial^{2} \phi\right]\right\|_{L_{t}^{q} L^{p^{\prime}}} \\
& \leq C\left\||\nabla|^{\alpha+3-4 / p} \phi\right\|_{L^{\infty} L^{2}}\|\partial \phi\|_{L^{2} L^{p}}\|\partial \phi\|_{L^{p / 2} L^{2 p /(p-4)}} \\
& \quad+C\left\||\nabla|^{\alpha+2-4 / p} \phi\right\|_{L^{\infty} L^{2}}\left\|\partial^{2} \phi\right\|_{L^{2} L^{p}}\|\partial \phi\|_{L^{p / 2} L^{2 p /(p-4)}} .
\end{aligned}
$$

Now, since $\alpha \leq 2+3 / p$,

$$
\begin{aligned}
& \left\||\nabla|^{\alpha+3-4 / p} \phi\right\|_{L^{\infty} L^{2}}+\left\||\nabla|^{\alpha+2-4 / p} \phi\right\|_{L^{\infty} L^{2}} \\
& \quad \leq C\|(f, g)\|_{H^{5-1 / p} \times H^{4-1 / p}}\|\partial \phi\|_{L^{2} L^{p}}+\left\|\partial^{2} \phi\right\|_{L^{2} L^{p}} \leq C X_{T}(\phi) .
\end{aligned}
$$

Regarding the term $\|\partial \phi\|_{L^{p / 2} L^{2 p /(p-4)}}$, we observe that $(2 / p,(p-4) /(2 p))=$ $4 / p(1 / 2,0)+(1-4 / p)(0,1 / 2)$ and thus, by Gagliardo-Nirenberg's inequality and the energy estimates of Proposition 3.1,

$$
\begin{aligned}
\|\partial \phi\|_{L^{p / 2} L^{2 p /(p-4)}} & \leq\|\partial \phi\|_{L^{2} L^{\infty}}^{4 / p}\|\partial \phi\|_{L^{\infty} L^{2}}^{1-4 / p} \\
& \leq C X_{T}(\phi)^{4 / p}\|(f, g)\|_{\dot{H}^{1} \times L^{2}}^{1-4 / p} \\
& \leq X_{T}(\phi)+C_{p}\|(f, g)\|_{\dot{H}^{1} \times L^{2}},
\end{aligned}
$$


where in the last line, we have used Young's inequality. Putting everything together, we arrive at

$$
\begin{aligned}
& X_{T}(\phi) \leq C\|(f, g)\|_{W^{4-1 / p, p^{\prime}} \times W^{3-1 / p, p^{\prime}}}+\|(f, g)\|_{H^{7 / 2} \times H^{5 / 2}} \\
& +C\|(f, g)\|_{H^{5-1 / p} \times H^{4-1 / p}} X_{T}(\phi)\left(X_{T}(\phi)+C_{p}\|(f, g)\|_{\dot{H}^{1} \times L^{2}}\right) .
\end{aligned}
$$

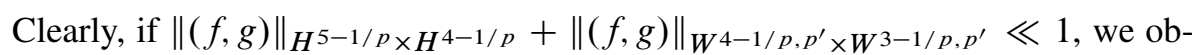
tain

$$
X_{T}(\phi) \leq C_{p}\left(\|(f, g)\|_{H^{5-1 / p} \times H^{4-1 / p}}+\|(f, g)\|_{W^{4-1 / p, p^{\prime}} \times W^{3-1 / p, p^{\prime}}}\right),
$$

which is the desired control of $X_{T}(\phi)$.

\section{A Proof of Lemma 3.4}

The proof of Lemma 3.4 follows standard techniques. According to the definition of Err ${ }^{k}$ (taking into account $\|\nabla \phi\|_{L^{2} L^{\infty}}\left\|\nabla^{2} \phi\right\|_{L^{2} L^{\infty}} \leq \varepsilon^{2}$ ), we obtain

$$
\begin{aligned}
& \sum_{k=-\infty}^{\infty} 2^{2 k s}\left\|\operatorname{Err}^{k}(t)\right\|_{L^{1}[0, T] L^{2}}^{2} \\
& \quad \leq C_{n} \varepsilon^{4} \sum_{k=-\infty}^{\infty} 2^{2 k(s-1)}\left(\sum_{l>k-5}\left\|\nabla_{x t}^{2} \phi_{l}\right\|_{L^{\infty}[0, T] L^{2}}\right)^{2} \\
& \quad=C_{n} \varepsilon^{4} \sum_{k=-\infty}^{\infty} 2^{2 k(s-1)} \sum_{l_{1}, l_{2}>k-5}\left\|\nabla_{x t}^{2} \phi_{l_{1}}\right\|_{L^{\infty}[0, T] L^{2}}\left\|\nabla_{x t}^{2} \phi_{l_{2}}\right\|_{L^{\infty}[0, T] L^{2}} \\
& \leq C_{n} \varepsilon^{4} \sum_{l_{1}, l_{2}} 2^{2 \min \left(l_{1}, l_{2}\right)(s-1)}\left\|\nabla_{x t}^{2} \phi_{l_{1}}\right\|_{L^{\infty}[0, T] L^{2}}\left\|\nabla_{x t}^{2} \phi_{l_{2}}\right\|_{L^{\infty}[0, T] L^{2},}
\end{aligned}
$$

where in the last step, we have used that since $s>1$

$$
\sum_{k \leq \min \left(l_{1}, l_{2}\right)+5} 2^{2 k(s-1)} \lesssim 2^{2 \min \left(l_{1}, l_{2}\right)(s-1)} .
$$

We bound the last double sum by

$$
\begin{gathered}
\left(\sum_{l_{1}, l_{2}} 2^{2 \min \left(l_{1}, l_{2}\right)(s-1)}\left\|\nabla_{x t}^{2} \phi_{l_{1}}\right\|_{L^{\infty}[0, T] L^{2}}^{2}\right)^{1 / 2} \\
\quad \times\left(\sum_{l_{1}, l_{2}} 2^{2 \min \left(l_{1}, l_{2}\right)(s-1)}\left\|\nabla_{x t}^{2} \phi_{l_{2}}\right\|_{L^{\infty}[0, T] L^{2}}^{2}\right)^{1 / 2} \\
\leq C \sum_{l} 2^{2 l(s-1)}\left\|\nabla_{x t}^{2} \phi_{l}\right\|_{L^{\infty}[0, T] L^{2}}^{2} \leq C \sum_{l} \tilde{E}_{l}(s, T)
\end{gathered}
$$




\section{B Proof of Lemma 3.5}

By the definition of $\tilde{E}_{k}(s, t)$, we have to estimate $\sum_{k} 2^{2 k(s-1)}\left\|\partial_{t t}^{2} \phi_{k}\right\|_{L^{\infty}[0, T] L^{2}}^{2}$. Recall (3.6). Thus, for classical solutions we can take Littlewood-Paley projections of (3.6) and estimate as follows:

$$
\begin{aligned}
& 2^{2 k(s-1)}\left\|\partial_{t t}^{2} \phi_{k}\right\|_{L^{\infty}[0, T] L^{2}}^{2} \\
& \leq C 2^{2 k(s+1)}\left\|\phi_{k}\right\|_{L^{\infty}[0, T] L^{2}}^{2}+C 2^{2 k(s-1)}\left\|A(\phi)\left[\nabla_{t x} \nabla_{x} \phi\right]_{>k-5}\right\|_{L^{\infty}[0, T] L^{2}}^{2} \\
& \quad+C 2^{2 k(s-1)}\left\|A(\phi)_{\sim k}\left[\nabla_{t x} \nabla_{x} \phi\right]_{\leq k-5}\right\|_{L^{\infty}[0, T] L^{2}}^{2} \\
& \quad+C 2^{2 k(s-1)}\left\|B(\phi)\left[\partial_{t t}^{2} \phi\right]_{>k-5}\right\|_{L^{\infty}[0, T] L^{2}}^{2} \\
& \quad+C 2^{2 k(s-1)}\left\|B(\phi)_{\sim k}\left[\partial_{t t}^{2} \phi\right]_{\leq k-5}\right\|_{L^{\infty}[0, T] L^{2}}^{2} .
\end{aligned}
$$

We easily have by Hölder's inequality and elementary manipulations of sums,

$$
\begin{gathered}
\sum_{k} 2^{2 k(s-1)}\left\|A(\phi)\left[\nabla_{t x} \nabla_{x} \phi\right]_{>k-5}\right\|_{L^{\infty}[0, T] L^{2}}^{2} \leq\|A(\phi)\|_{L_{t x}^{\infty}}^{2} \sum_{k} E_{k}(s, T) \\
\sum_{k} 2^{2 k(s-1)}\left\|B(\phi)\left[\partial_{t t}^{2} \phi\right]_{>k-5}\right\|_{L^{\infty}[0, T] L^{2}}^{2} \\
\leq\|B(\phi)\|_{L_{t x}^{\infty}}^{2} \sum_{l} 2^{2 l(s-1)}\left\|\partial_{t t}^{2} \phi_{l}\right\|_{L^{\infty}[0, T] L^{2}}^{2}
\end{gathered}
$$

This step takes care of these two terms, since

$$
\|A(\phi)\|_{L_{t x}^{\infty}}+\|B(\phi)\|_{L_{t x}^{\infty}} \leq C\|\nabla \phi\|_{L_{t x}^{\infty}}^{2}
$$

For the other two terms, we have

$$
\begin{aligned}
& \sum_{k} 2^{2 k(s-1)}\left[\left\|A(\phi)_{\sim k}\left[\nabla_{t x} \nabla_{x} \phi\right]_{\leq k-5}\right\|_{L^{\infty}[0, T] L^{2}}^{2}\right. \\
& \leq C\left\|\nabla_{t x} \nabla_{x} \phi\right\|_{L^{\infty} L^{n}}^{2} \sum_{k} 2^{2 k(s-1)}\left[\left\|[\partial \phi \partial \phi]_{k}\right\|_{L^{\infty} L^{2 n /(n-2)}}^{2}\right. \\
& \sum_{k} 2^{2 k(s-1)}\left\|B(\phi)_{\sim k}\left[\partial_{t t}^{2} \phi\right]_{\leq k-5}\right\|_{\left.L^{\infty}[0, T] L^{2}\right]}^{2} \\
& \leq C\left\|\partial_{t t}^{2} \phi\right\|_{L^{\infty} L^{n}}^{2} \sum_{k} 2^{2 k(s-1)}\left[\left\|[\partial \phi \partial \phi]_{k}\right\|_{L^{\infty} L^{2 n /(n-2)}}^{2}\right.
\end{aligned}
$$


A quick computation using (3.6) shows that

$$
\begin{gathered}
\left\|\partial_{t t}^{2} \phi\right\|_{L^{\infty} L^{n}} \leq\|\Delta \phi\|_{L^{\infty} L^{n}}+\|A(\phi)\|_{L_{t x}^{\infty}}\left\|\nabla_{t x} \nabla_{x} \phi\right\|_{L^{\infty} L^{n}} \\
+\|B(\phi)\|_{L_{t x}^{\infty}}\left\|\partial_{t t}^{2} \phi\right\|_{L^{\infty} L^{n}} \\
\leq C\left(1+\|\nabla \phi\|_{L_{t x}^{\infty}}^{2}\left\|\nabla_{t x} \nabla_{x} \phi\right\|_{L^{\infty} L^{n}}\right. \\
+C\|\nabla \phi\|_{L_{t x}^{\infty}}^{2}\left\|\partial_{t t}^{2} \phi\right\|_{L^{\infty} L^{n}}
\end{gathered}
$$

and hence for $\|\nabla \phi\|_{L_{t x}^{\infty}} \leq \varepsilon$, so that $C \varepsilon<1 / 2$, we easily conclude

$$
\left\|\partial_{t t}^{2} \phi\right\|_{L^{\infty} L^{n}} \leq C\left\|\nabla_{t x} \nabla_{x} \phi\right\|_{L^{\infty} L^{n}} .
$$

In addition, we will show that

$$
\sum_{k} 2^{2 k(s-1)}\left[\left\|[\partial \phi \partial \phi]_{k}\right\|_{L^{\infty} L^{2 n /(n-2)}}^{2} \leq C\left\|\nabla_{x t} \phi\right\|_{L_{t x}^{\infty}}^{2} \sum_{k} E_{k}(s, T) .\right.
$$

Let us finish the proof of Lemma 3.5, assuming the validity of (B.2). Indeed, combining all estimates for $\sum_{k} 2^{2 k(s-1)}\left\|\partial_{t t}^{2} \phi_{k}\right\|_{L^{\infty}[0, T] L^{2}}^{2}$ yields

$$
\begin{aligned}
\sum_{k} \tilde{E}_{k}(s, T) \leq C \sum_{k} E_{k}(s, T)+C\left\|\nabla_{t x} \phi\right\|_{L_{t x}^{\infty}}^{4} \sum_{k} \tilde{E}_{k}(s, T) \\
+C\left\|\nabla_{t x} \nabla_{x} \phi\right\|_{L^{\infty} L^{n}}^{2}\left\|\nabla_{t x} \phi\right\|_{L_{t x}^{\infty}}^{2} \cdot \sum_{k} \tilde{E}_{k}(s, T) .
\end{aligned}
$$

Since $\left\|\nabla_{t x} \phi\right\|_{L_{t x}^{\infty}} \leq \varepsilon$ and $\left\|\nabla_{t x} \nabla_{x} \phi\right\|_{L^{\infty} L^{n}} \leq C\left\|\nabla_{t x} \phi\right\|_{L^{\infty} \dot{H}^{n / 2}} \leq C \varepsilon$, we conclude

$$
\sum_{k} \tilde{E}_{k}(s, T) \leq C \sum_{k} E_{k}(s, T)
$$

\section{B.1 Proof of (B.2)}

Following the standard decomposition,

$$
\begin{aligned}
P_{k}[u v] & =P_{k}\left[u_{\leq k-5} v_{\sim k}\right]+\sum P_{k}\left[u_{k-5 \leq-\leq k+5} v\right]+P_{k}\left[u_{>k+5} v\right] \\
& =P_{k}\left[u_{\leq k-5} v_{\sim k}\right]+\sum P_{k}\left[u_{k-5 \leq-\leq k+5} v\right]+\sum_{l>k+5} P_{k}\left[u_{l} v_{l-2 \leq-\leq l+2}\right] .
\end{aligned}
$$


For the first term, we have by Hölder's and Bernstein's inequality

$$
\begin{aligned}
\sum_{k} 2^{2 k(s-1)}\left\|\partial \phi_{\leq k-5} \partial \phi_{\sim k}\right\|_{L^{\infty} L^{2 n /(n-2)}}^{2} & \\
& \leq\|\nabla \phi\|_{L_{t x}^{\infty}}^{2} \sum_{k} 2^{2 k(s-1)}\left\|\partial \phi_{\sim k}\right\|_{L^{\infty} L^{2 n /(n-2)}}^{2} \\
& \leq C\|\nabla \phi\|_{L_{t x}^{\infty}}^{2} \sum_{k} 2^{2 k s}\|\partial \phi \sim k\|_{L^{\infty} L^{2}}^{2} \\
& \leq C\left\|\nabla_{x t} \phi\right\|_{L_{t x}^{\infty}}^{2} \sum_{k} E_{k}(s, T) .
\end{aligned}
$$

The second term is treated in a similar fashion:

$$
\begin{aligned}
\sum_{k} 2^{2 k(s-1)}\left\|\partial \phi_{k-5 \leq \cdot \leq k+5} \partial \phi\right\|_{L^{\infty} L^{2 n /(n-2)}}^{2} & \\
& \leq C\|\partial \phi\|_{L_{t x}^{\infty}}^{2} \sum_{k} 2^{2 k(s-1)}\left\|\partial \phi_{k-5 \leq-\leq k+5}\right\|_{L^{\infty} L^{2 n /(n-2)}}^{2} \\
& \leq C\left\|\nabla_{x t} \phi\right\|_{L_{t x}^{\infty}}^{2} \sum_{k} E_{k}(s, T) .
\end{aligned}
$$

For the third term,

$$
\begin{aligned}
\sum_{k} 2^{2 k(s-1)}\left[\sum_{l>k+5}\left\|\partial \phi_{l} \partial \phi_{l-2 \leq \cdot \leq l+2}\right\|_{L^{\infty} L^{2 n /(n-2)}}\right]^{2} \\
\leq C_{s} \sum_{l_{1}, l_{2}} 2^{2 \min \left(l_{1}, l_{2}\right)(s-1)}\left\|\partial \phi_{l_{1}} \partial \phi_{l_{1}-2 \leq \leq l_{1}+2}\right\|_{L^{\infty} L^{2 n /(n-2)}} \\
\quad \times\left\|\partial \phi_{l_{2}} \partial \phi_{l_{2}-2 \leq \cdot \leq l_{2}+2}\right\|_{L^{\infty} L^{2 n /(n-2)}} \\
\leq C_{s} \sum_{l} 2^{2 l(s-1)}\left\|\partial \phi_{l} \partial \phi_{l-2 \leq-\leq l+2}\right\|_{L^{\infty} L^{2 n /(n-2)}}^{2} \\
\leq C_{s}\|\nabla \phi\|_{L_{t x}^{\infty}}^{2} \sum_{l} 2^{2 l(s-1)}\left\|\partial \phi_{l}\right\|_{L^{\infty} L^{2 n /(n-2)}}^{2} \\
\leq C_{s}\|\nabla \phi\|_{L_{t x}^{\infty}}^{2} \sum_{l} E_{l}(s, T) .
\end{aligned}
$$




\section{Proof of Lemma 3.6}

We start with an useful and elementary lemma, concerning null forms.

Lemma C.1. Let $T>0$ and $u, v, w \in C^{\infty}\left([0, T] \times \mathbb{R}^{n}\right)$ be smooth functions. Then

$$
\begin{aligned}
& \int_{0}^{T} \int Q(u, v) w d x d t \\
& \quad=\int_{0}^{T} \int(-v Q(u, w) d x d t+v w \square u) d x d t+\left.\int v u_{t} w\right|_{t=0} ^{T} d x
\end{aligned}
$$

The verification of Lemma C.1 is straightforward and thus omitted. Apply now Lemma C. 1 for the triple $u=\phi_{<k-10}, v=Q\left(\phi_{<k-10}, \phi_{k}\right), w=\partial_{t} \phi_{k}$. We get

$$
\begin{aligned}
\left.\int_{0}^{T} \int_{\left(\phi_{<}-10\right.}, Q\left(\phi_{<k-10}, \phi_{k}\right)\right) \partial_{t} \phi_{k} d x d t & \\
=- & \int_{0}^{T} \int Q\left(\phi_{<k-10}, \phi_{k}\right) Q\left(\phi_{<k-10}, \partial_{t} \phi_{k}\right) d x d t \\
& +\int_{0}^{T} \int Q\left(\phi_{<k-10}, \phi_{k}\right) \partial_{t} \phi_{k} \square \phi_{<k-10} d x d t \\
& +\int^{1}\left[\left.Q\left(\phi_{<k-10}, \phi_{k}\right) \partial_{t} \phi_{<k-10} \partial_{t} \phi_{k}\right|_{t=0} ^{T}\right] d x \\
=-\frac{\int_{0}}{2} \int_{t}\left[Q\left(\phi_{<k-10}, \phi_{k}\right)\right]^{2} d x d t & \\
& +\int_{0}^{T} \int Q\left(\phi_{<k-10}, \phi_{k}\right) Q\left(\partial_{t} \phi_{<k-10}, \phi_{k}\right) d x d t \\
& +\int_{0}^{T} \int Q\left(\phi_{<k-10}, \phi_{k}\right) \partial_{t} \phi_{k} \square \phi_{<k-10} d x d t \\
& +\int^{T}\left[\left.Q\left(\phi_{<k-10}, \phi_{k}\right) \partial_{t} \phi_{<k-10} \partial_{t} \phi_{k}\right|_{t=0} ^{T}\right] d x .
\end{aligned}
$$

Take absolute values and note that the first term integrates in time. Also note

$$
\begin{aligned}
& \left|\int\left[\left.Q\left(\phi_{<k-10}, \phi_{k}\right) \partial_{t} \phi_{<k-10} \partial_{t} \phi_{k}\right|_{t=0} ^{T}\right] d x\right| \\
& \leq C\left\|\nabla_{t, x} \phi_{k}\right\|_{L^{\infty}[0, T] L^{2}}^{2}\left\|\nabla_{t, x} \phi\right\|_{L^{\infty}[0, T] L^{\infty}}^{2}
\end{aligned}
$$


All together, by Hölder's inequality,

$$
\begin{aligned}
&\left|\int_{0}^{T} \int Q\left(\phi_{<k-10}, Q\left(\phi_{<k-10}, \phi_{k}\right)\right) \partial_{t} \phi_{k} d x d t\right| \\
& \leq \sup _{0 \leq t \leq T}\left|\int\left[Q\left(\phi_{<k-10}, \phi_{k}\right)\right]^{2} d x\right| \\
&+\left|\int Q\left(\phi_{<k-10}, \phi_{k}\right) Q\left(\partial_{t} \phi_{<k-10}, \phi_{k}\right) d x d t\right| \\
&+\left|\int Q\left(\phi_{<k-10}, \phi_{k}\right) \partial_{t} \phi_{k} \square \phi_{<k-10} d x d t\right| \\
&+C\left\|\nabla_{t, x} \phi_{k}\right\|_{L^{\infty}[0, T] L^{2}}^{2}\left\|\nabla_{t, x} \phi\right\|_{L^{\infty}[0, T] L^{\infty}}^{2} \\
& \leq C\left\|\nabla_{t, x} \phi_{k}\right\|_{L^{\infty}[0, T] L^{2}}^{2}\left\|\nabla_{t, x} \phi\right\|_{L^{\infty}[0, T] L^{\infty}}^{2} \\
&+C\left\|\nabla_{x t}^{2} \phi\right\|_{L^{2} L^{\infty}}\left\|\nabla_{x t} \phi\right\|_{L^{2} L^{\infty}\left\|\nabla_{x t} \phi_{k}\right\|_{L^{\infty}[0, T] L^{2}}^{2}}
\end{aligned}
$$

which is, of course, the claim of Lemma 2.2.

\section{Proof of Lemma 4.1}

First of all, by Sobolev embedding and the formulas (2.2), (2.3) and scale invariance, matters reduce to checking the claims in Lemma 4.1 for $\alpha=0$ and a single frequency component $w_{k}$ of the solution, corresponding to a fixed frequency data $F_{k}$. Thus, setting $F$ to be Fourier supported in a fixed annulus $\left\{|\xi| \sim 2^{k}\right\}$, we will need to show

$$
\left\|w_{k}\right\|_{L^{2} L^{p}} \leq C_{p} 2^{k(1-4 / p)}\left\|F_{k}\right\|_{L_{t}^{q} L^{p^{\prime}}}
$$

For this purpose, we use the decay estimate (2.8) with $f=0, g$ (i.e. for $z=$ $\left.\frac{\sin (t \sqrt{-\Delta})}{\sqrt{-\Delta}} g\right)$

$$
\left\|\frac{\sin (t \sqrt{-\Delta})}{\sqrt{-\Delta}} g\right\|_{L_{x}^{\infty}} \leq \frac{C}{t}\||\nabla| g\|_{L_{x}^{1}}
$$

and the energy estimate $\left\|\frac{\sin (t \sqrt{-\Delta})}{\sqrt{-\Delta}} g\right\|_{L_{x}^{2}} \leq\left\||\nabla|^{-1} g\right\|_{L^{2}}$. Interpolating between the two estimates yields

$$
\left\|\frac{\sin (t \sqrt{-\Delta})}{\sqrt{-\Delta}} g\right\|_{L_{x}^{p}} \leq \frac{C}{t^{1-2 / p}}\left\||\nabla|^{1-4 / p} g\right\|_{L^{p^{\prime}}} .
$$


Thus, for our problem in Lemma 4.1, we have

$$
w(t, \cdot)=\int_{0}^{t} \frac{\sin ((t-s) \sqrt{-\Delta})}{\sqrt{-\Delta}} F(s, \cdot) d s
$$

and hence an application of (D.1) yields

$$
\|w\|_{L^{p}} \leq C \int_{0}^{t} \frac{\left\||\nabla|^{1-4 / p} F(s, \cdot)\right\|_{L^{p^{\prime}}}}{|t-s|^{1-2 / p}} d s .
$$

Take the $L_{t}^{2}$ norm of the last expression. By the Hardy-Littlewood-Sobolev inequality, and since $p>4$,

$$
\begin{aligned}
\|w\|_{L^{2} L^{p}} & \leq C\left\||t-s|^{-(1-2 / p)}\right\|_{L^{p /(p-2), \infty}}\left\||\nabla|^{1-4 / p} F(s, \cdot)\right\|_{L^{q} L_{x}^{p^{\prime}}} \\
& \leq C\left\||\nabla|^{1-4 / p} F(s, \cdot)\right\|_{L^{q} L_{x}^{p^{\prime}}},
\end{aligned}
$$

where $q$ satisfies $1 / q=1 / 2+2 / p$.

\section{Bibliography}

[1] S. Alinhac and P. Gérard, Pseudo-differential Operators and the Nash-Moser Theorem, Graduate Studies in Mathematics 82, American Mathematical Society, Providence, RI, 2007.

[2] P. Allen, L. Andersson and J. Isenberg, Timelike minimal submanifolds of general co-dimension in Minkowski space time, J. Hyperbolic Differ. Equ. 3 (2006), no. 4, 691-700.

[3] D. Chae and H. Huh, Global existence for small initial data in the Born-Infeld equations, J. Math. Phys. 44 (2003), no. 12, 6132-6139.

[4] J. Hoppe, Some classical solutions of relativistic membrane equations in 4-spacetime dimensions, Phys. Lett. B 329 (1994), no. 1, 10-14.

[5] L. Hörmander, Lectures on Nonlinear Hyperbolic Differential Equations, Mathematics \& Applications 26, Springer-Verlag, Berlin, 1997.

[6] T. Kato and G. Ponce, Commutator estimates and the Euler and Navier-Stokes equations, Comm. Pure Appl. Math. 41 (1988), no. 7, 891-907.

[7] M. Keel and T.Tao, Endpoint Strichartz estimates, Amer. J. Math 120 (1998), 955980.

[8] S. Klainerman, Global existence for nonlinear wave equations, Comm. Pure Appl. Math. 33 (1980), no. 1, 43-101. 
[9] D. Kong, Q. Sun and Yi Zhou, The equation for time-like extremal surfaces in Minkowski space $\mathbb{R}^{2+n}$, J. Math. Phys. 47 (2006), no. 1, 013503, 16 pp.

[10] H. Lindblad, A remark on global existence for small initial data of the minimal surface equation in Minkowskian spaces-time, Proc. Amer. Math. Soc. 132 (2004), no. 4, 1095-1102

[11] J. Liu and Yi Zhou, Asymptotic behaviour of global classical solutions of diagonalizable quasilinear hyperbolic systems, Math. Methods Appl. Sci. 30 (2007), no. 4, 479-500.

[12] J. Liu and Yi Zhou, Initial-boundary value problem for the equation of timelike extremal surfaces in Minkowski space, J. Math. Phys. 49 (2008), no. 4, 043507.

[13] C. Sogge, Lectures on Nonlinear Wave Equations, Monographs in Analysis II, International Press, Boston, MA, 1995.

[14] T. Tao, Global regularity of wave maps I. Small critical Sobolev norm in high dimension, IMRN 7 (2001), 299-328.

[15] D. Tataru, Strichartz estimates for second order hyperbolic operators with nonsmooth coefficients. III, J. Amer. Math. Soc. 15 (2002), no. 2, 419-442.

Received October 13, 2008; revised May 11, 2009.

\section{Author information}

Atanas Stefanov, Department of Mathematics, University of Kansas, 1460, Jayhawk

Blvd, Lawrence, KS 66044, USA.

E-mail: stefanov@math.ku . edu 
Copyright of Forum Mathematicum is the property of De Gruyter and its content may not be copied or emailed to multiple sites or posted to a listserv without the copyright holder's express written permission. However, users may print, download, or email articles for individual use. 